\title{
Crystal Engineering and Photophysical Properties of Phenyl-Pyrenoimidazole Systems
}

Zahra A. Tabasi, Joshua C. Walsh, Graham J. Bodwell, David W. Thompson, and Yuming Zhao*

Department of Chemistry, Memorial University, St. John's, Newfoundland and Labrador, CANADA A1B 3X7; yuming@mun.ca

\section{Table of Content}

1. Synthetic Procedures for Compounds 3a-h

S2

2. NMR Spectra of Compounds 3a-e and $3 f$

S6

3. DFT and TD-DFT Computational Results

S23 


\section{Synthetic and Characterization of Compounds 3a-h}

The synthesis and spectroscopic characterization of compounds $\mathbf{3} \mathbf{f}$ and $\mathbf{3 g}$ were reported in our previous publication. ${ }^{1}$ The synthesis of compounds $\mathbf{3 a}, \mathbf{3 e}$, and $\mathbf{3 h}$ were previously reported by Karthik et $a l .^{2}$ and their NMR and MS characterization data are consistent with our experimental results.

\section{Compound 3a}

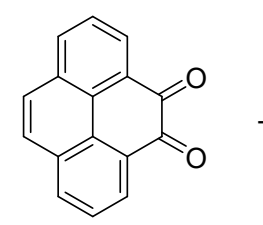

1

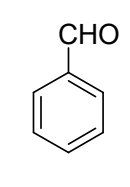

$2 a$

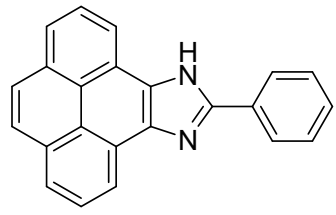

3a

Pyrene-4,5-dione (1) (0.200 g, $0.861 \mathrm{mmol})$, benzaldehyde (2a) (0.274 g, $2.58 \mathrm{mmol})$, ammonium acetate $(1.33 \mathrm{~g}, 17.2 \mathrm{mmol})$, and glacial acetic acid $(99.7 \%, 7 \mathrm{~mL})$ were mixed in a round-bottom flask equipped with a condenser. The reaction was heated at $110{ }^{\circ} \mathrm{C}$ for $12 \mathrm{~h}$, and then slowly cooled down to rt. The resulting precipitate was collected by vacuum filtration and the filtrate was sequentially washed with glacial acetic acid, satd $\mathrm{NaHCO}_{3}$ solution (aq), and water to yield crude product 3a. The crude product was further purified by silica flash column chromatography using ethyl acetate/hexanes $(1: 4, \mathrm{v} / \mathrm{v})$ as eluent to afford pure compound $3 \mathbf{a}$ ( $0.170 \mathrm{~g}, 0.534 \mathrm{mmol}, 62 \%$ yield) as an off-white solid. ${ }^{1} \mathrm{H}$ NMR (300 MHz, DMSO- $\left.d_{6}\right): \delta 13.75$ (s, $1 \mathrm{H}), 8.85(\mathrm{~d}, J=7.4 \mathrm{~Hz}, 2 \mathrm{H}), 8.43-8.38(\mathrm{~m}, 2 \mathrm{H}), 8.27$ (dd, $J=7.7,1.1 \mathrm{~Hz}, 2 \mathrm{H}), 8.22-8.13$ (m, 4H), $7.66(\mathrm{t}, J=7.5 \mathrm{~Hz}, 2 \mathrm{H}), 7.54(\mathrm{t}, J=7.3 \mathrm{~Hz}, 1 \mathrm{H}) ;{ }^{13} \mathrm{C}$ NMR $\left(75 \mathrm{MHz}\right.$, DMSO- $\left.d_{6}\right): \delta$ 149.90, 131.99, 130.86, 129.81, 129.48, 128.16 (br), 126.77, 126.69, 124.62, 122.25, 119.47 (two carbon signals missing due to coincidental overlap); FTIR (neat): 3044, 3052, 2920, 2851, $1599,1552,1456,1160,825,704,689 \mathrm{~cm}^{-1}$; HRMS (APPI-TOF, positive mode) $\mathrm{m} / \mathrm{z}$ : calcd for $\mathrm{C}_{23} \mathrm{H}_{14} \mathrm{~N}_{2}[\mathrm{M}+\mathrm{H}]^{+}, 319.1157$; found 319.1226; X-ray.

\section{Compound 3b}
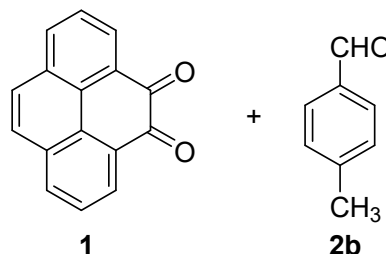

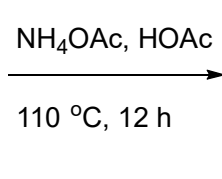

2b

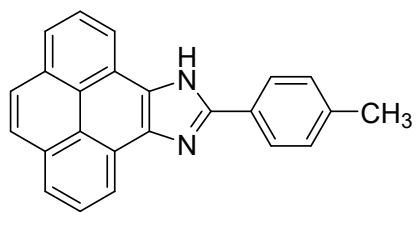

$3 b$

\footnotetext{
${ }^{1}$ Tabasi, Z. A.; Younes, E. A.; Walsh, J. C.; Thompson, D. W.; Bodwell, G. J.; Zhao, Y. ACS Omega 2018, 3, 16387-6397.

${ }^{2}$ Karthik, S.; Ajantha, J.; Nagaraja, C. M.; Easwaramoorthi, S.; Gandhi, T. Org. Biomol. Chem. 2016, 14, 1025510266.
} 
Pyrene-4,5-dione (1) (0.200 g, $0.861 \mathrm{mmol}), p$-methylbenzaldehyde (2b) $(0.310 \mathrm{~g}, 2.58 \mathrm{mmol})$, ammonium acetate $(1.33 \mathrm{~g}, 17.2 \mathrm{mmol})$, and glacial acetic acid $(99.7 \%, 7 \mathrm{~mL})$ were mixed in a round-bottom flask equipped with a condenser. The reaction was heated at $110{ }^{\circ} \mathrm{C}$ for $12 \mathrm{~h}$, and then slowly cooled down to rt. The resulting precipitate was collected by vacuum filtration and the filtrate was sequentially washed with glacial acetic acid, satd $\mathrm{NaHCO}_{3}$ solution (aq), and water to yield crude product 3a. The crude product was further purified by silica flash column chromatography using ethyl acetate/hexanes $(2: 98, \mathrm{v} / \mathrm{v})$ as eluent to afford pure compound $\mathbf{3 b}$ ( $0.137 \mathrm{~g}, 0.413 \mathrm{mmol}, 48 \%$ yield) as a yellow solid. ${ }^{1} \mathrm{H}$ NMR (300 MHz, DMSO- $\left.d_{6}\right): \delta 13.66$ (s, $1 \mathrm{H}), 8.83(\mathrm{t}, J=6.6 \mathrm{~Hz}, 2 \mathrm{H}), 8.36-8.08(\mathrm{~m}, 8 \mathrm{H}), 7.46(\mathrm{~d}, J=8.0 \mathrm{~Hz}, 2 \mathrm{H}), 2.44(\mathrm{~s}, 3 \mathrm{H}) ;{ }^{13} \mathrm{C}$ NMR (75 MHz, DMSO- $\left.d_{6}\right)$ : $\delta 150.07,139.45,132.07,131.91,129.99,128.35,128.17,127.94$, $126.84,126.65,124.59,124.48,122.26,122.20,122.13,120.85,119.49,119.35,21.50$ (two carbon signals missing due to coincidental overlap); FTIR (neat): 3605, 3008, 2916, 1662, 1603, $1555,1432,1258,823,712,691 \mathrm{~cm}^{-1}$; HRMS (APPI-TOF, positive mode) $\mathrm{m} / z$ : calcd for $\mathrm{C}_{24} \mathrm{H}_{16} \mathrm{~N}_{2}[\mathrm{M}+\mathrm{H}]^{+}$, 333.1313; found 333.1379; X-ray.

\section{Compound 3c}

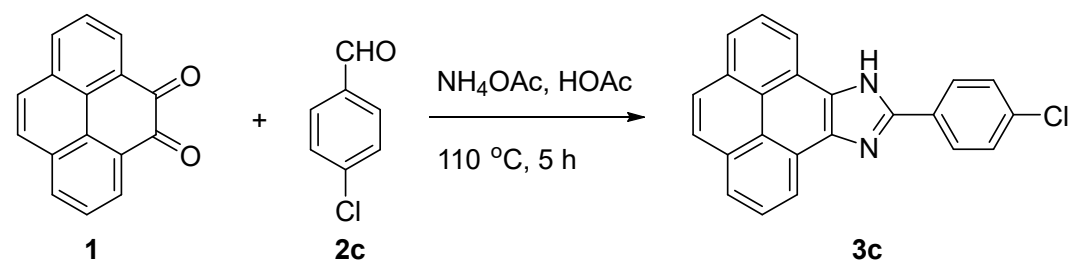

Pyrene-4,5-dione (1) (0.200 g, $0.861 \mathrm{mmol}), p$-chlorobenzaldehyde (2c) $(0.363 \mathrm{~g}, 2.58 \mathrm{mmol})$, ammonium acetate $(1.33 \mathrm{~g}, 17.2 \mathrm{mmol})$, and glacial acetic acid $(99.7 \%, 7 \mathrm{~mL})$ were mixed in a round-bottom flask equipped with a condenser. The reaction was heated at $110{ }^{\circ} \mathrm{C}$ for $5 \mathrm{~h}$, and then slowly cooled down to rt. The resulting precipitate was collected by vacuum filtration and the filtrate was sequentially washed with glacial acetic acid, satd $\mathrm{NaHCO}_{3}$ solution (aq), and water to yield crude product 3c. The crude product was further purified by silica flash column chromatography using ethyl acetate/hexanes $(2: 98, \mathrm{v} / \mathrm{v})$ as eluent to afford pure compound $\mathbf{3 c}$ $\left(0.173 \mathrm{~g}, 0.491 \mathrm{mmol}, 57 \%\right.$ yield) as an off-white solid. ${ }^{1} \mathrm{H}$ NMR (300 MHz, DMSO- $\left.d_{6}\right): \delta 13.82$ (s, $1 \mathrm{H}), 8.82(\mathrm{~d}, J=7.3 \mathrm{~Hz}, 2 \mathrm{H}), 8.41(\mathrm{~d}, J=8.7 \mathrm{~Hz}, 2 \mathrm{H}), 8.27(\mathrm{~d}, J=7.7 \mathrm{~Hz}, 2 \mathrm{H}), 8.22-8.13$ (m, 4H), $7.73(\mathrm{~d}, J=8.7 \mathrm{~Hz}, 2 \mathrm{H}) ;{ }^{13} \mathrm{C}$ NMR $\left(75 \mathrm{MHz}\right.$, DMSO- $\left.d_{6}\right): \delta 148.76,134.35,132.00$, $129.69,129.59,128.33,126.81,124.76,122.30,119.48$ (three carbon signals missing due to coincidental overlap); FTIR (neat): 3627, 3043, 2920, 2851, 1682, 1633, 1551, 1433, 1179, 824, 712, $568 \mathrm{~cm}^{-1}$; HRMS (APPI-TOF, positive mode) $\mathrm{m} / z$ : calcd for $\mathrm{C}_{23} \mathrm{H}_{13} \mathrm{~N}_{2}{ }^{35} \mathrm{Cl}[\mathrm{M}+\mathrm{H}]^{+}$, 353.0767 found 353.0859; X-ray.

\section{Compound 3d}




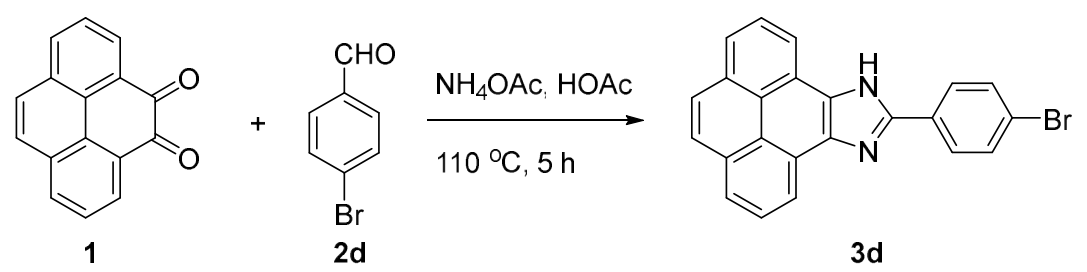

Pyrene-4,5-dione (1) (0.200 g, $0.861 \mathrm{mmol}), p$-bromobenzaldehyde (2d) $(0.477 \mathrm{~g}, 2.58 \mathrm{mmol})$, ammonium acetate $(1.33 \mathrm{~g}, 17.2 \mathrm{mmol})$, and glacial acetic acid $(99.7 \%, 7 \mathrm{~mL})$ were mixed in a round-bottom flask equipped with a condenser. The reaction was heated at $110{ }^{\circ} \mathrm{C}$ for $5 \mathrm{~h}$, and then slowly cooled down to rt. The resulting precipitate was collected by vacuum filtration and the filtrate was sequentially washed with glacial acetic acid, satd $\mathrm{NaHCO}_{3}$ solution (aq), and water to yield crude product 3d. The crude product was further purified by silica flash column chromatography using acetone/hexanes $(1: 99, \mathrm{v} / \mathrm{v})$ as eluent to afford pure compound 3d $(0.150$ g, $0.378 \mathrm{mmol}, 44 \%$ yield) as a light brown solid. ${ }^{1} \mathrm{H}$ NMR (300 MHz, DMSO- $\left.d_{6}\right): \delta 13.83$ (s, $1 \mathrm{H}), 8.82(\mathrm{~m}, 2 \mathrm{H}), 8.34(\mathrm{~d}, J=8.7 \mathrm{~Hz}, 2 \mathrm{H}), 8.30-8.24(\mathrm{~m}, 2 \mathrm{H}), 8.23-8.11(\mathrm{~m}, 4 \mathrm{H}), 7.86(\mathrm{~d}, J=$ $8.7 \mathrm{~Hz}, 2 \mathrm{H}) ;{ }^{13} \mathrm{C}$ NMR $\left(75 \mathrm{MHz}\right.$, DMSO- $\left.d_{6}\right): \delta 148.81,138.04,132.49,132.07,131.91,130.04$, $128.87,128.56,128.36,127.93,126.90,126.73,126.47,124.86,124.67,123.08,122.34,122.27$, 122.10, 119.52, 119.46; FTIR (neat): 3484, 3042, 2919, 2849, 1703, 1630, 1529, 1430, 1259, 1007, 711, 684, $562 \mathrm{~cm}^{-1}$; HRMS (APPI-TOF, positive mode) $\mathrm{m} / z$ : calcd for $\mathrm{C}_{23} \mathrm{H}_{13} \mathrm{~N}_{2}{ }^{79} \mathrm{Br}[\mathrm{M}$ $+\mathrm{H}]^{+}, 397.0262$ found 397.0407; X-ray.

\section{Compound 3e}
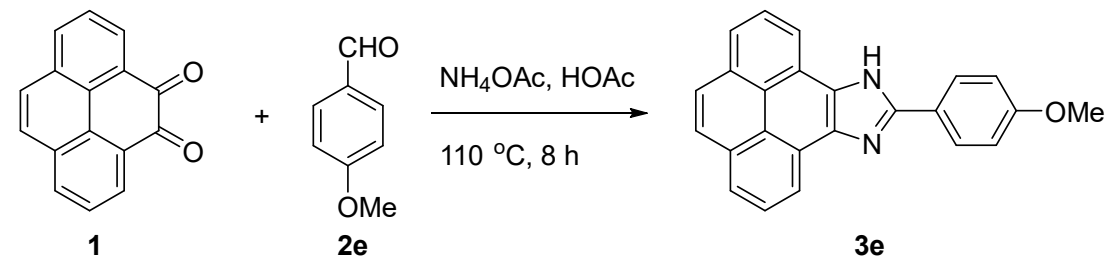

Pyrene-4,5-dione (1) (0.200 g, $0.861 \mathrm{mmol}), p$-methoxybenzaldehyde (2e) $(0.351 \mathrm{~g}, 2.58 \mathrm{mmol})$, ammonium acetate $(1.33 \mathrm{~g}, 17.2 \mathrm{mmol})$, and glacial acetic acid $(99.7 \%, 7 \mathrm{~mL})$ were mixed in a round-bottom flask equipped with a condenser. The reaction was heated at $110{ }^{\circ} \mathrm{C}$ for $8 \mathrm{~h}$, and then slowly cooled down to rt. The resulting precipitate was collected by vacuum filtration and the filtrate was sequentially washed with glacial acetic acid, satd $\mathrm{NaHCO}_{3}$ solution (aq), and water to yield crude product 3e. The crude product was further purified by silica flash column chromatography using acetone/hexanes $(5: 95, \mathrm{v} / \mathrm{v})$ as eluent to afford pure compound $\mathbf{3 e}$ $\left(0.138 \mathrm{~g}, 0.396 \mathrm{mmol}, 46 \%\right.$ yield) as a brown solid. ${ }^{1} \mathrm{H}$ NMR (300 MHz, DMSO- $\left.d_{6}\right): \delta 13.58$ (s, $1 \mathrm{H}), 8.82$ (dd, $J=9.9,7.7 \mathrm{~Hz}, 2 \mathrm{H}), 8.34$ (d, $J=8.9 \mathrm{~Hz}, 2 \mathrm{H}), 8.27-8.11(\mathrm{~m}, 6 \mathrm{H}), 7.22$ (d, $J=8.9$ $\mathrm{Hz}, 2 \mathrm{H}$ ), 3.89 (s, 3H); ${ }^{13} \mathrm{C}$ NMR (75 MHz, DMSO- $\left.d_{6}\right): \delta 160.74,150.07,137.91,132.13,132.07$, $131.91,128.39,128.34,128.26,127.93,126.80,126.65,126.59,124.42,124.40,123.51,122.21$, 122.02, 119.47, 119.38, 119.24, 119.20, 114.90, 55.84; FTIR (neat): 3308, 3000, 2957, 2851, 
1697, 1604, 1517, 1356, 1176, 824, 713,702 $\mathrm{cm}^{-1}$; HRMS (APPI-TOF, positive mode) $\mathrm{m} / \mathrm{z}$ : calcd for $\mathrm{C}_{24} \mathrm{H}_{16} \mathrm{~N}_{2} \mathrm{O}[\mathrm{M}+\mathrm{H}]^{+}, 349.1263$ found 349.1340; X-ray.

\section{Compound 3h}

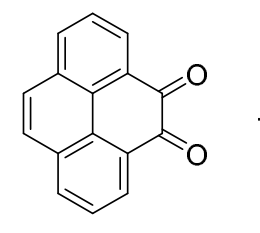

1

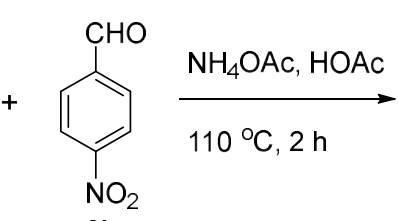

$2 \mathrm{~h}$

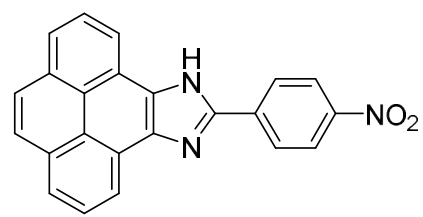

$3 \mathrm{~h}$

Pyrene-4,5-dione (1) (0.200 g, $0.861 \mathrm{mmol}), p$-nitrobenzaldehyde (2h) $(0.390 \mathrm{~g}, 2.58 \mathrm{mmol})$, ammonium acetate $(1.33 \mathrm{~g}, 17.2 \mathrm{mmol})$, and glacial acetic acid $(99.7 \%, 7 \mathrm{~mL})$ were mixed in a round-bottom flask equipped with a condenser. The reaction was heated at $110{ }^{\circ} \mathrm{C}$ for $8 \mathrm{~h}$, and then slowly cooled down to $\mathrm{rt}$. The resulting precipitate was collected by vacuum filtration and the filtrate was sequentially washed with glacial acetic acid, satd $\mathrm{NaHCO}_{3}$ solution (aq), and water to yield crude product $\mathbf{3 h}$. The crude product was further purified by silica flash column chromatography using acetone/hexanes $(5: 95, \mathrm{v} / \mathrm{v})$ as eluent to afford pure compound $\mathbf{3 h}(0.266$ g, $0.732 \mathrm{mmol}, 85 \%$ yield) as an orange colored solid. ${ }^{1} \mathrm{H}$ NMR (300 MHz, DMSO- $\left.d_{6}\right): \delta 14.13$ (s, 1H), $8.84(\mathrm{~d}, J=6.8 \mathrm{~Hz}, 2 \mathrm{H}), 8.62(\mathrm{~d}, J=9.0 \mathrm{~Hz}, 2 \mathrm{H}), 8.49$ (d, $J=9.0 \mathrm{~Hz}, 2 \mathrm{H}), 8.29$ (d, $J=$ $6.7 \mathrm{~Hz}, 2 \mathrm{H}), 8.22-8.14(\mathrm{~m}, 4 \mathrm{H}) ;{ }^{13} \mathrm{C}$ NMR $\left(75 \mathrm{MHz}, \mathrm{DMSO}-d_{6}\right): \delta 147.72,147.66,136.68$, $132.02,128.15,127.35,126.90,125.18,124.90,122.54,119.72$ (two carbon signals missing due

to coincidental overlap); FTIR (neat): 2920, 2851, 1595, 1486, 1343, 852, $709 \mathrm{~cm}^{-1}$; HRMS (APPI-TOF, positive mode) $m / z$ : calcd for $\mathrm{C}_{23} \mathrm{H}_{16} \mathrm{~N}_{3} \mathrm{O}_{2}[\mathrm{M}+\mathrm{H}]^{+}, 364.1008$ found 364.1080; Xray. 


\section{NMR Spectra of Compounds 3a-h}

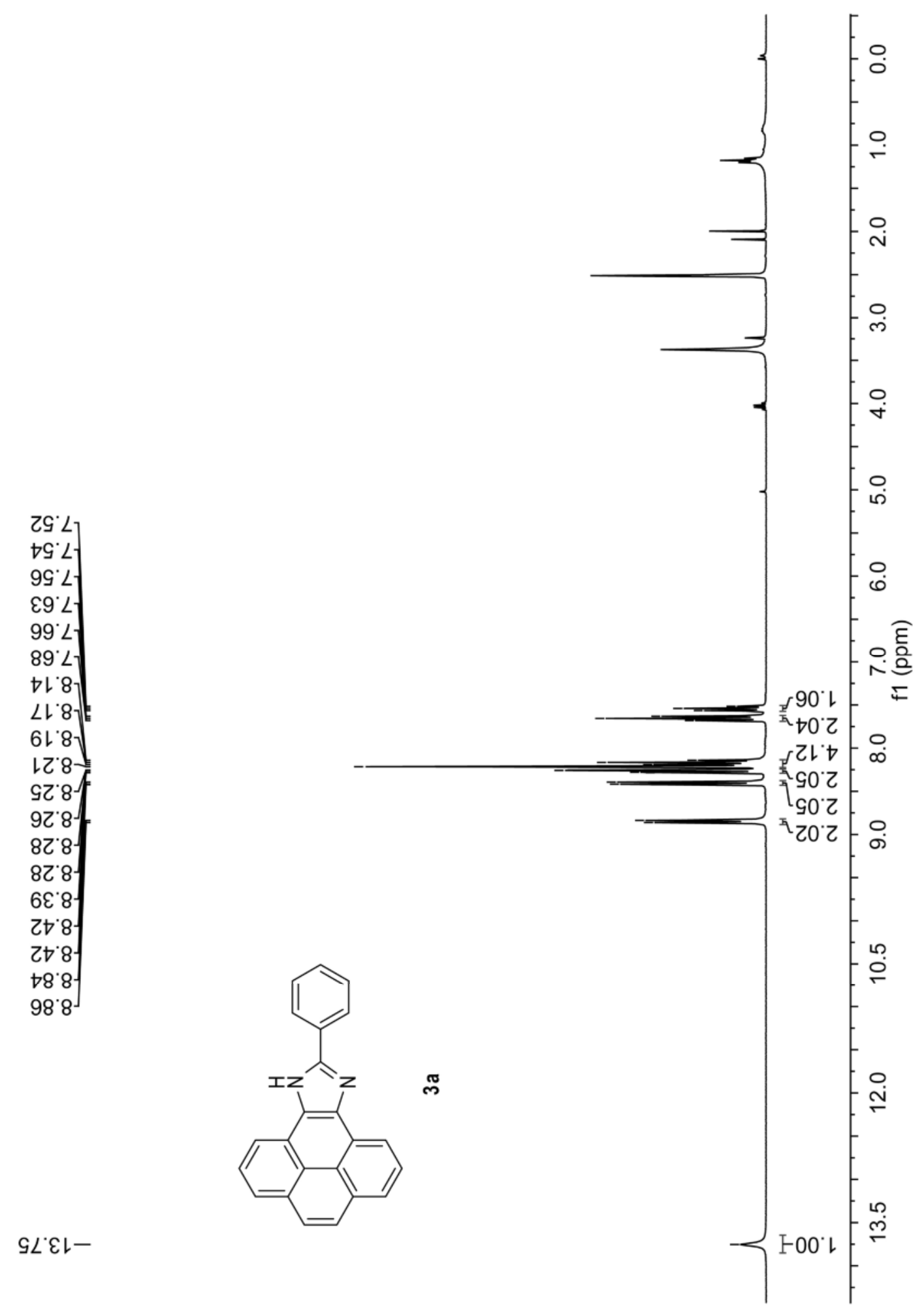

Fig. S-1 ${ }^{1} \mathrm{H}$ NMR $\left(300 \mathrm{MHz}\right.$, DMSO- $\left.d_{6}\right)$ spectrum of compound 3a. 


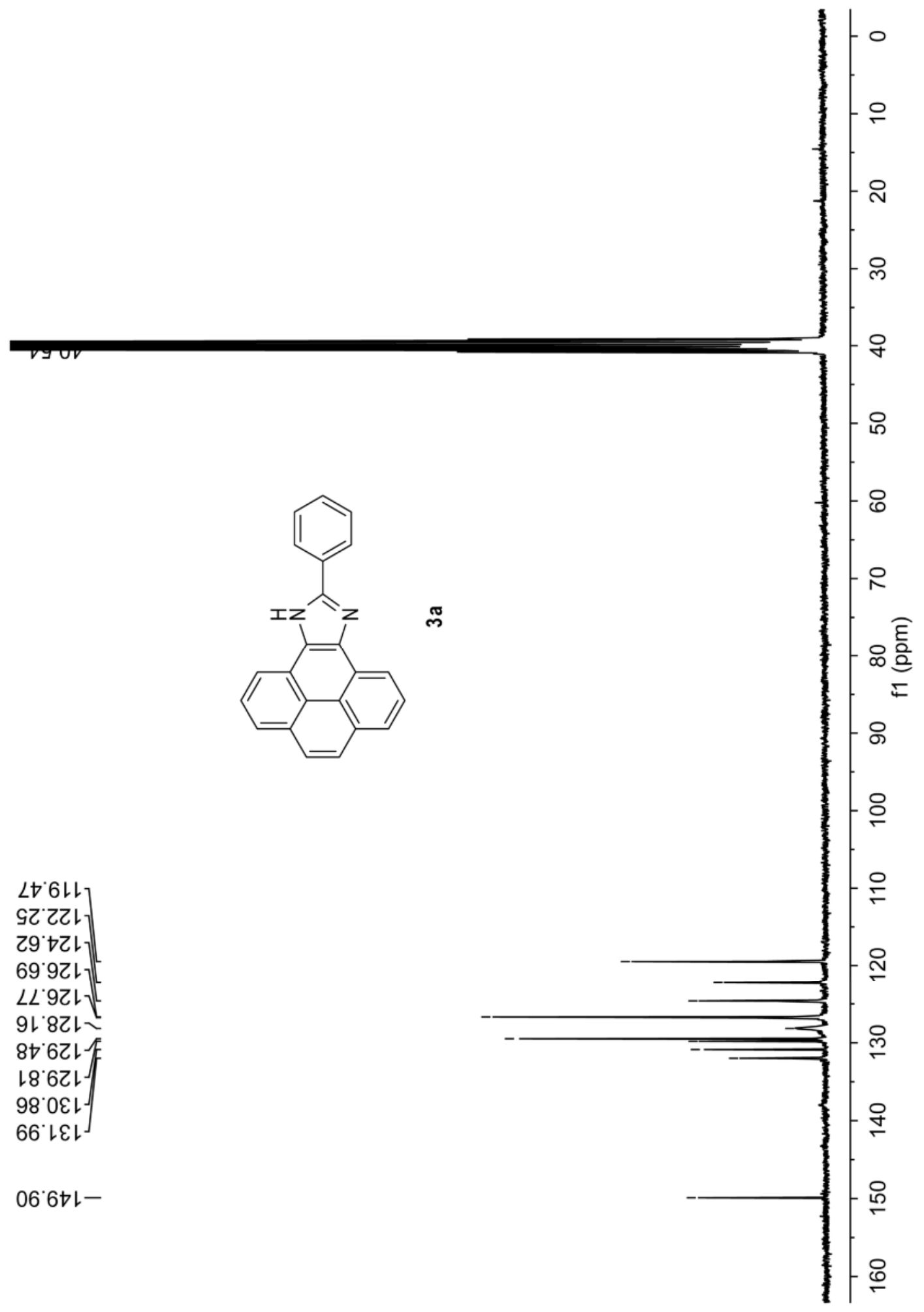

Fig. S-2 ${ }^{13} \mathrm{C}$ NMR (75 MHz, DMSO- $\left.d_{6}\right)$ spectrum of compound 3a. 
เซてー

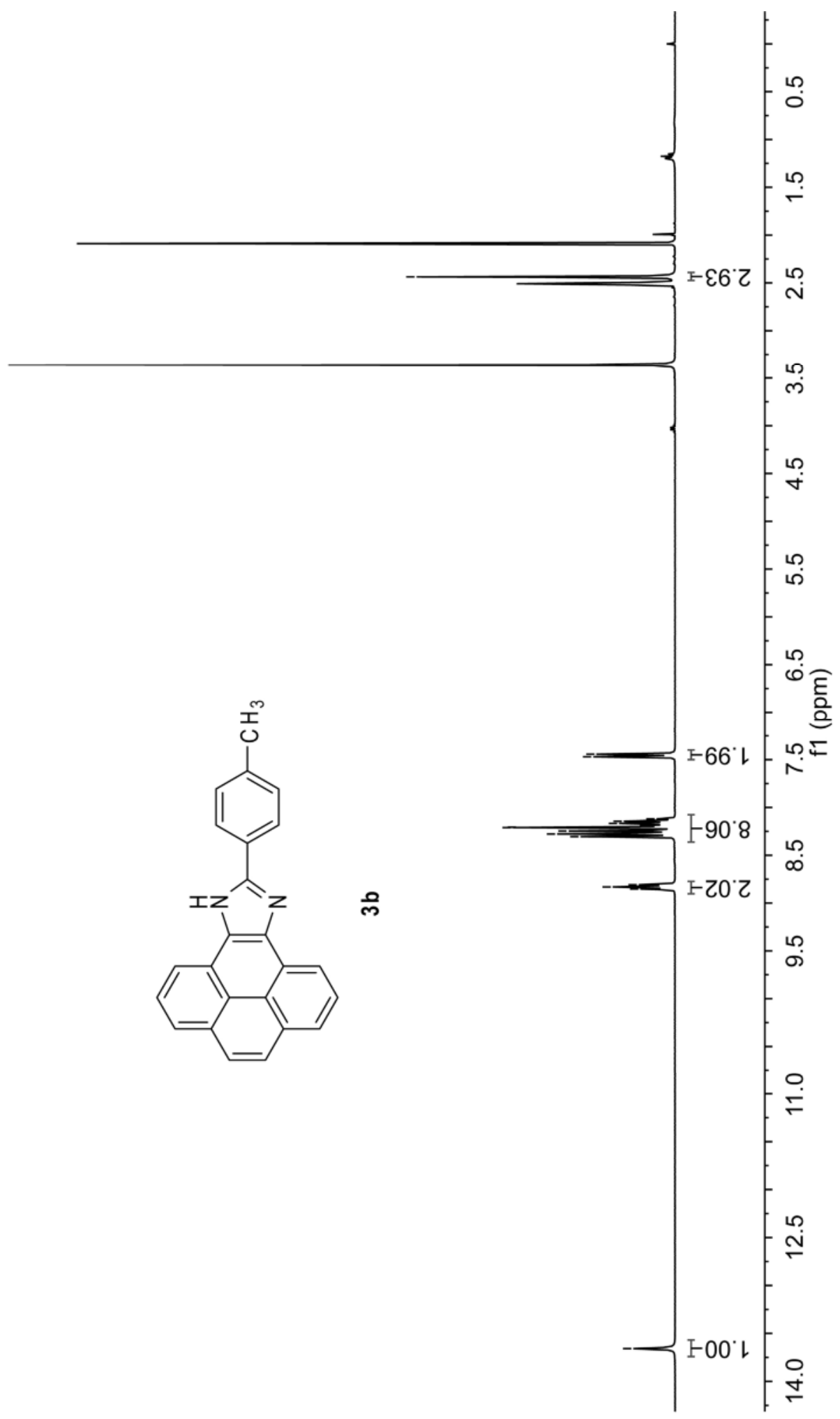

Fig. S-3 ${ }^{1} \mathrm{H}$ NMR (300 MHz, DMSO- $d_{6}$ ) spectrum of compound 3b. 

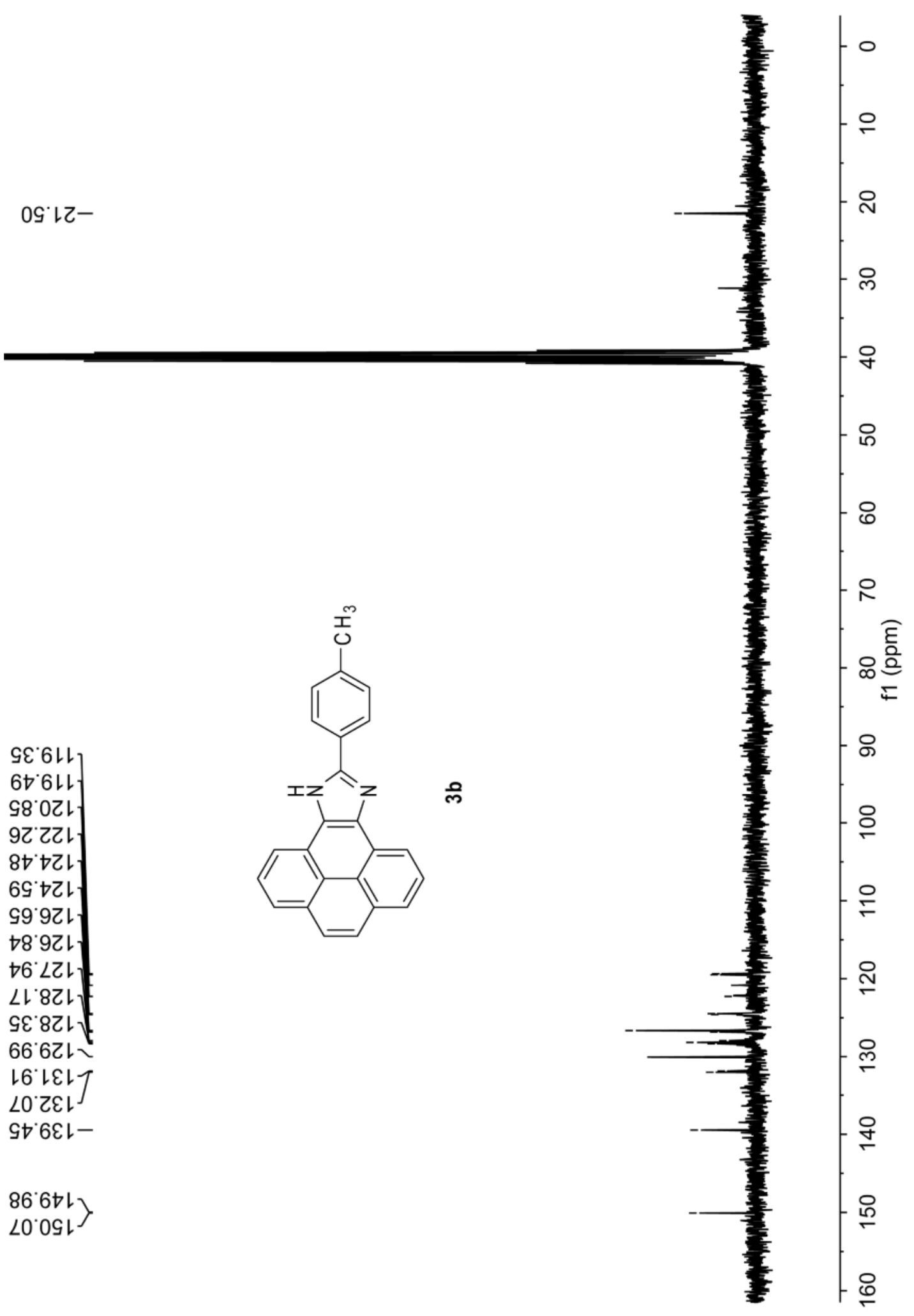

Fig. S-4 ${ }^{13} \mathrm{C}$ NMR (75 MHz, DMSO- $d_{6}$ ) spectrum of compound 3b. 


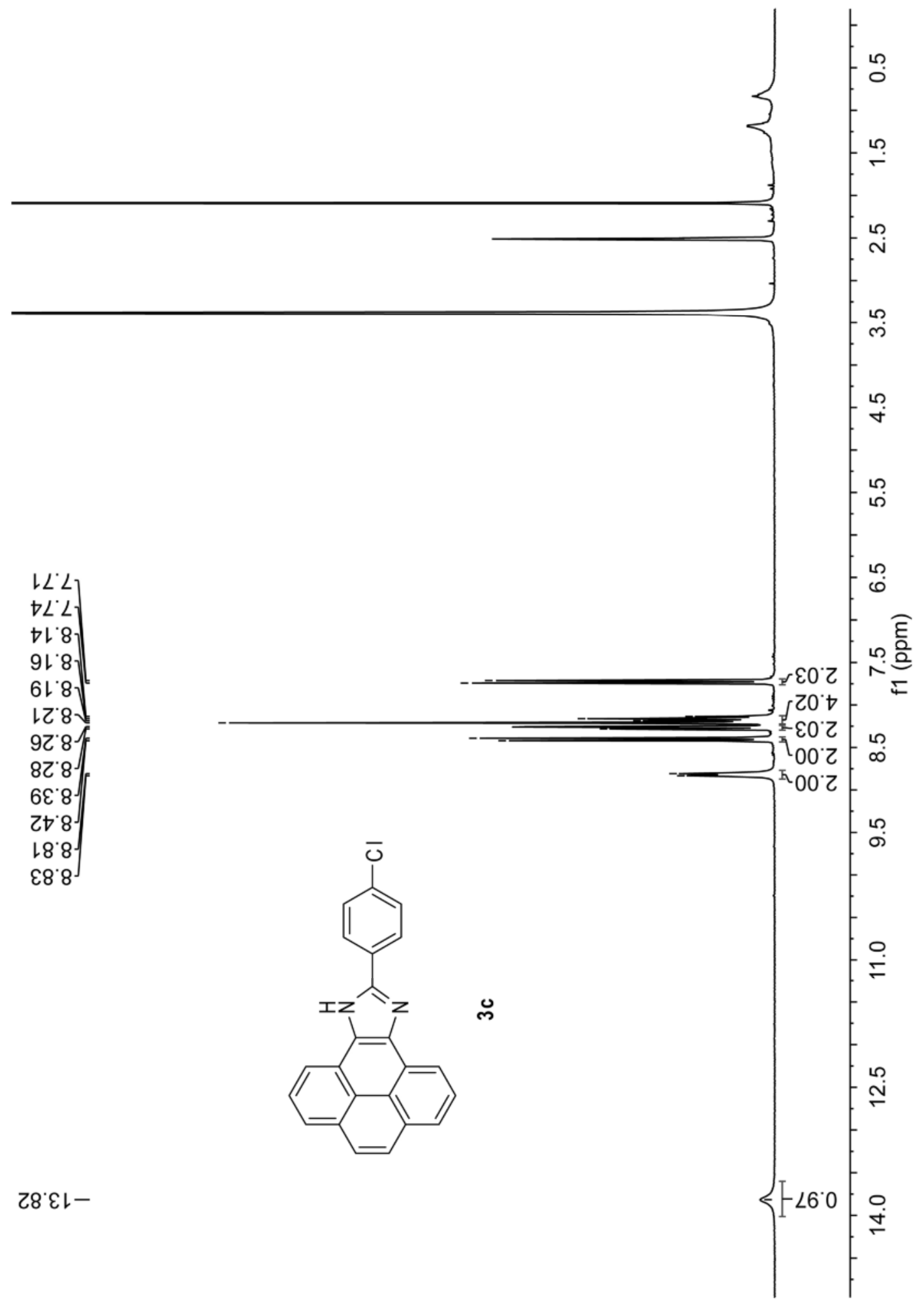

Fig. S-5 ${ }^{1} \mathrm{H}$ NMR (300 MHz, DMSO- $\left.d_{6}\right)$ spectrum of compound 3c. 


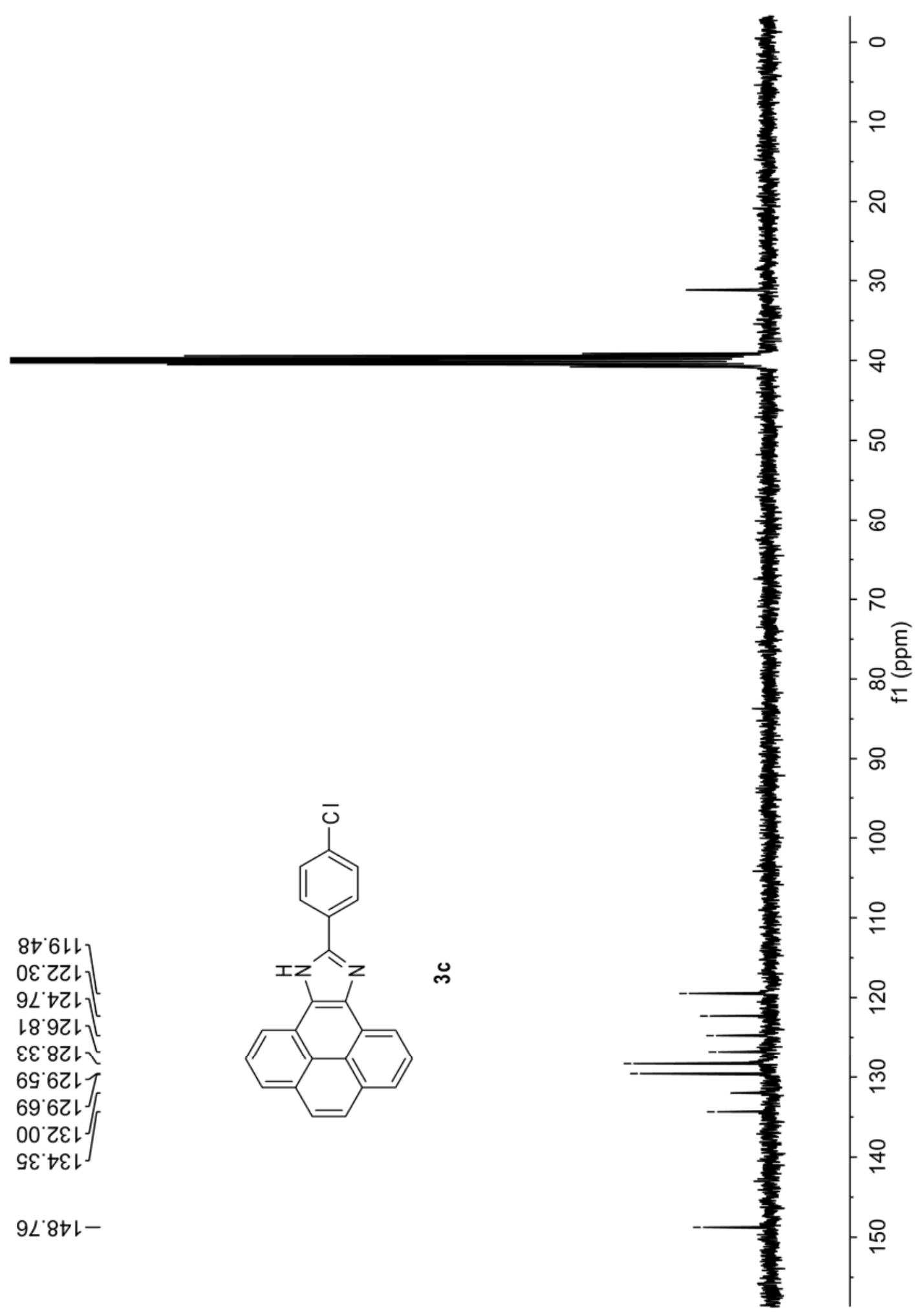

Fig. S-6 ${ }^{13} \mathrm{C}$ NMR (75 MHz, DMSO- $d_{6}$ ) spectrum of compound 3c. 


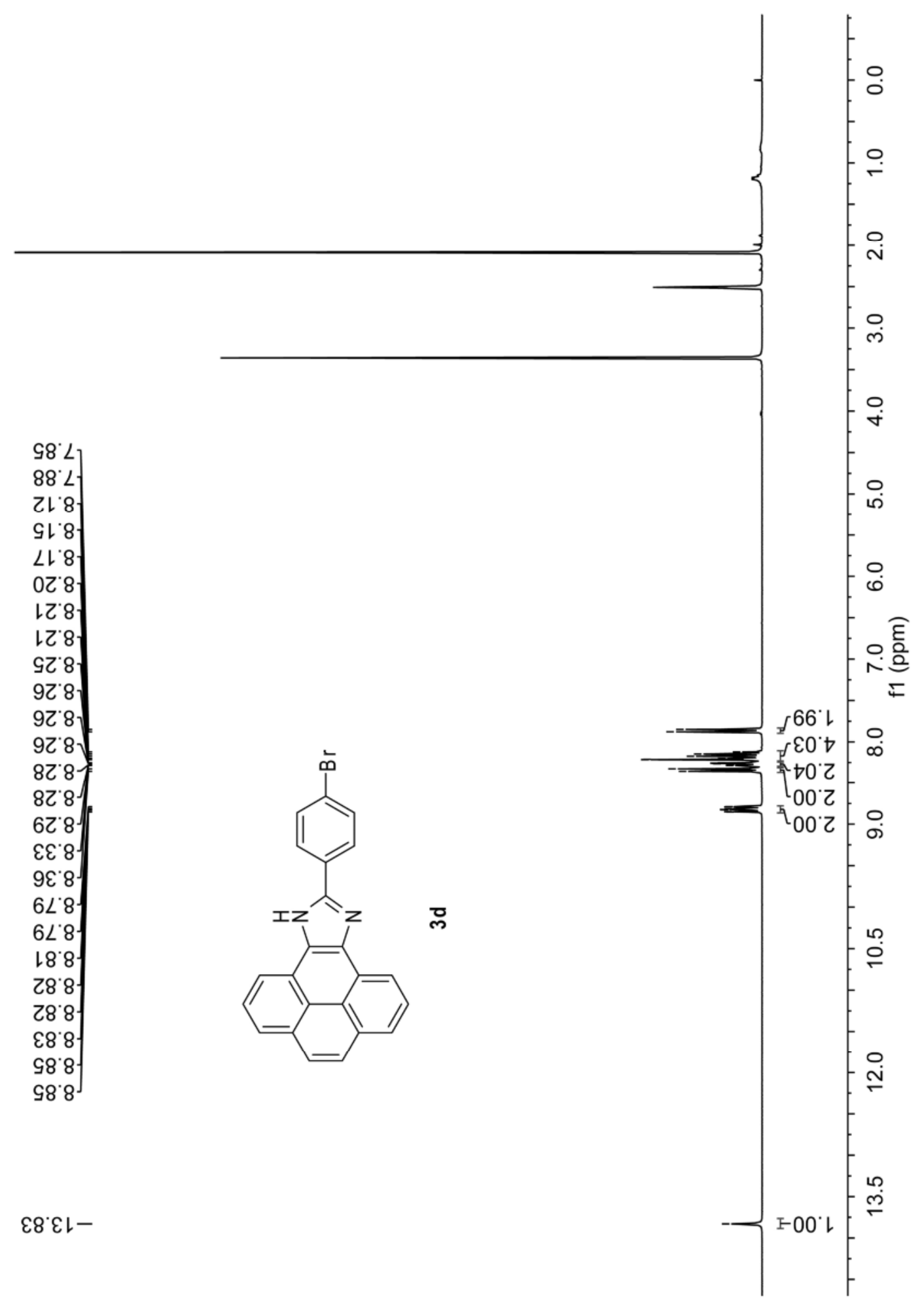

Fig. S-7 ${ }^{1} \mathrm{H}$ NMR (300 MHz, DMSO- $\left.d_{6}\right)$ spectrum of compound 3d. 


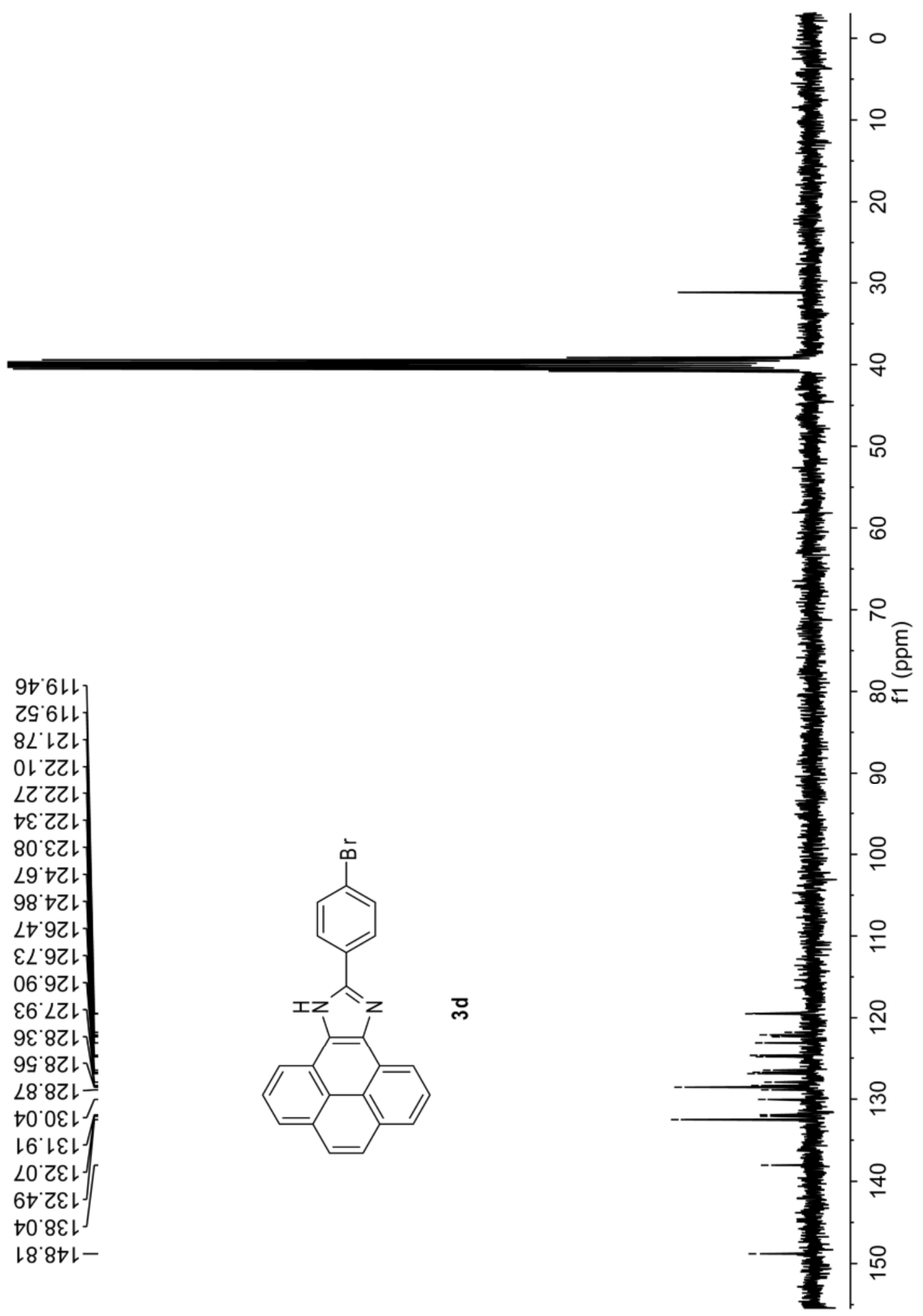

Fig. S-8 ${ }^{13} \mathrm{C}$ NMR (75 MHz, DMSO-d6) spectrum of compound 3d. 


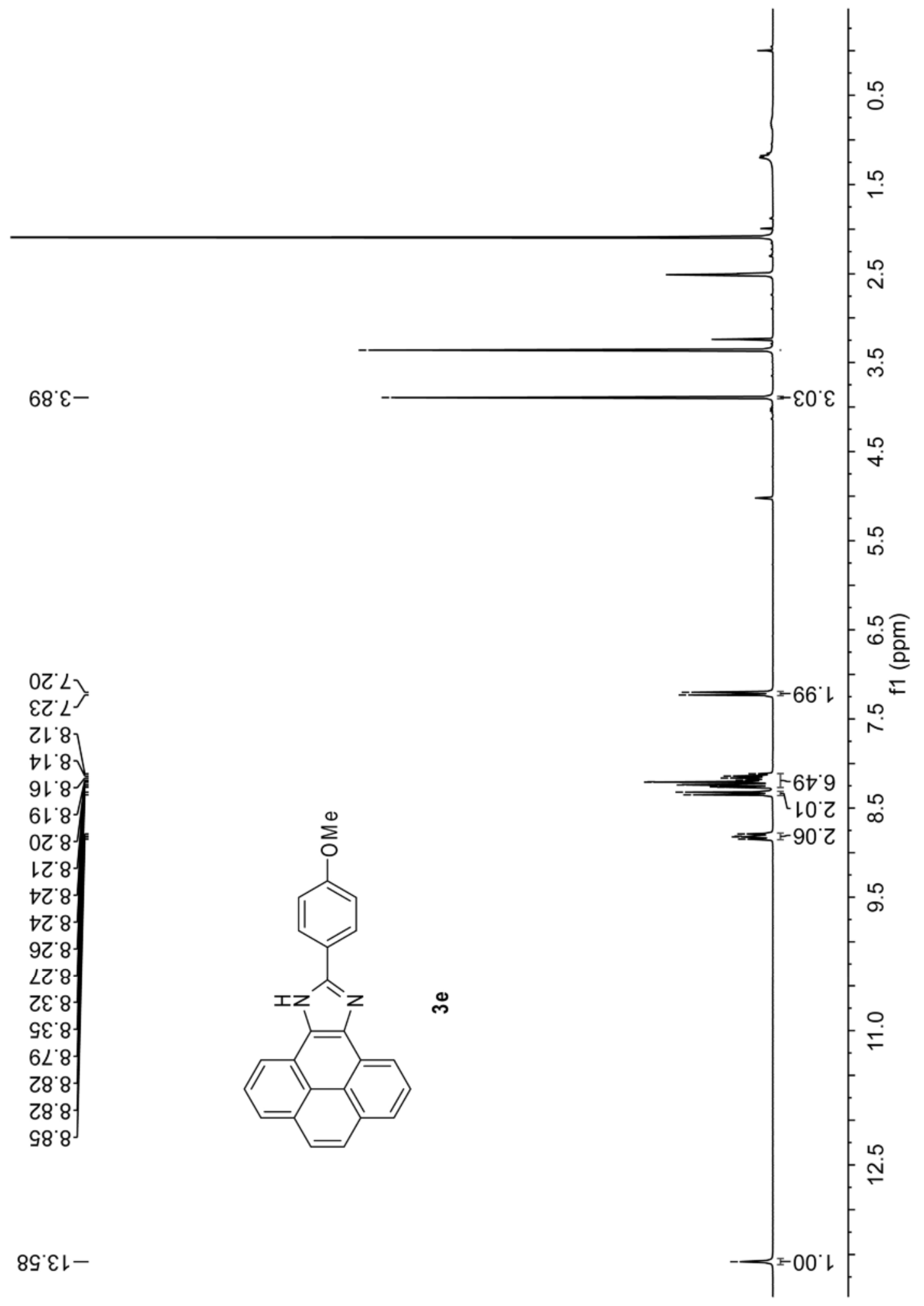

Fig. S-9 ${ }^{1} \mathrm{H}$ NMR $\left(300 \mathrm{MHz}, \mathrm{DMSO}-d_{6}\right)$ spectrum of compound 3e. 


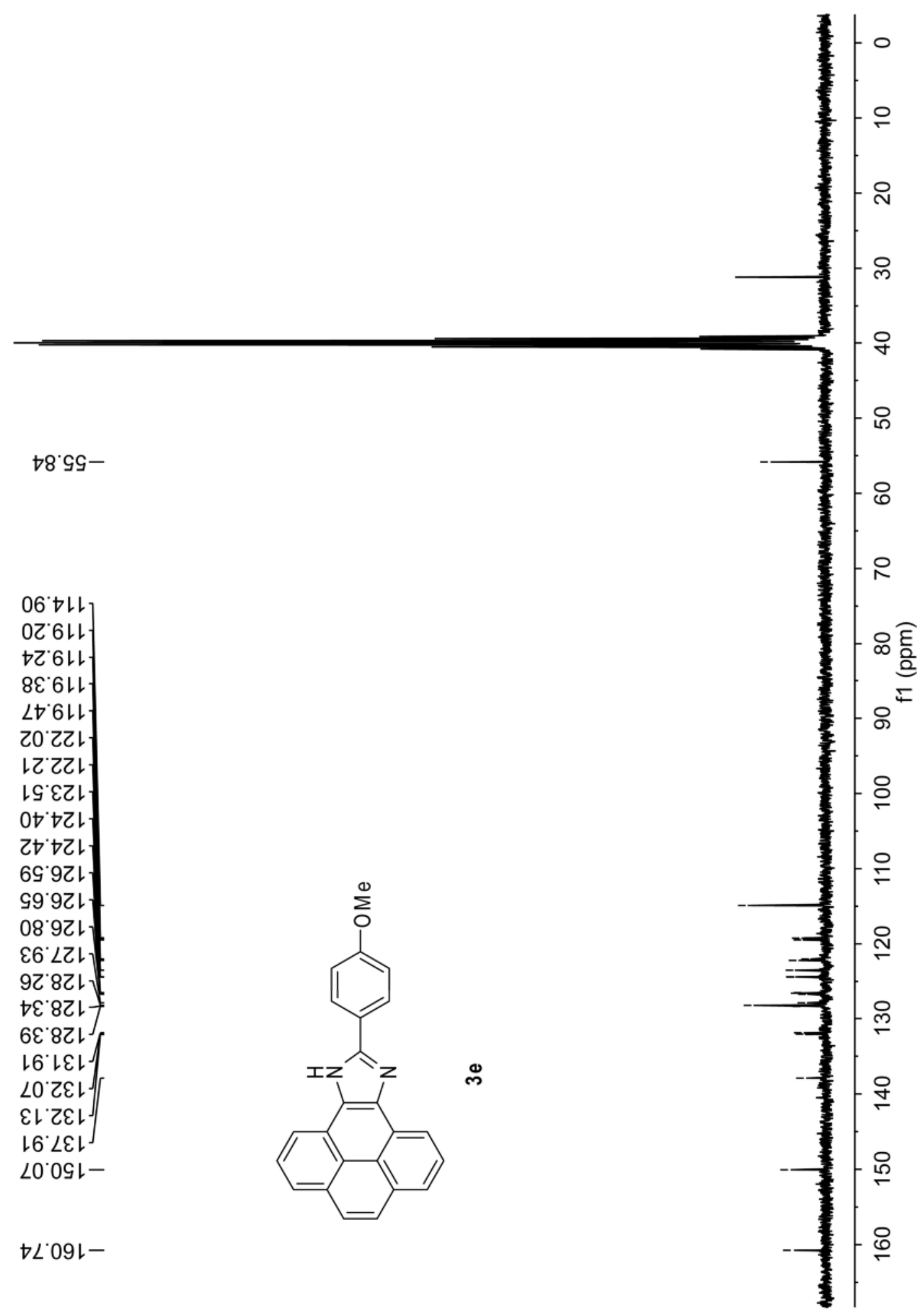

Fig. S-10 ${ }^{13} \mathrm{C}$ NMR (75 MHz, DMSO- $d_{6}$ ) spectrum of compound $3 \mathbf{e}$. 
Supporting Information for

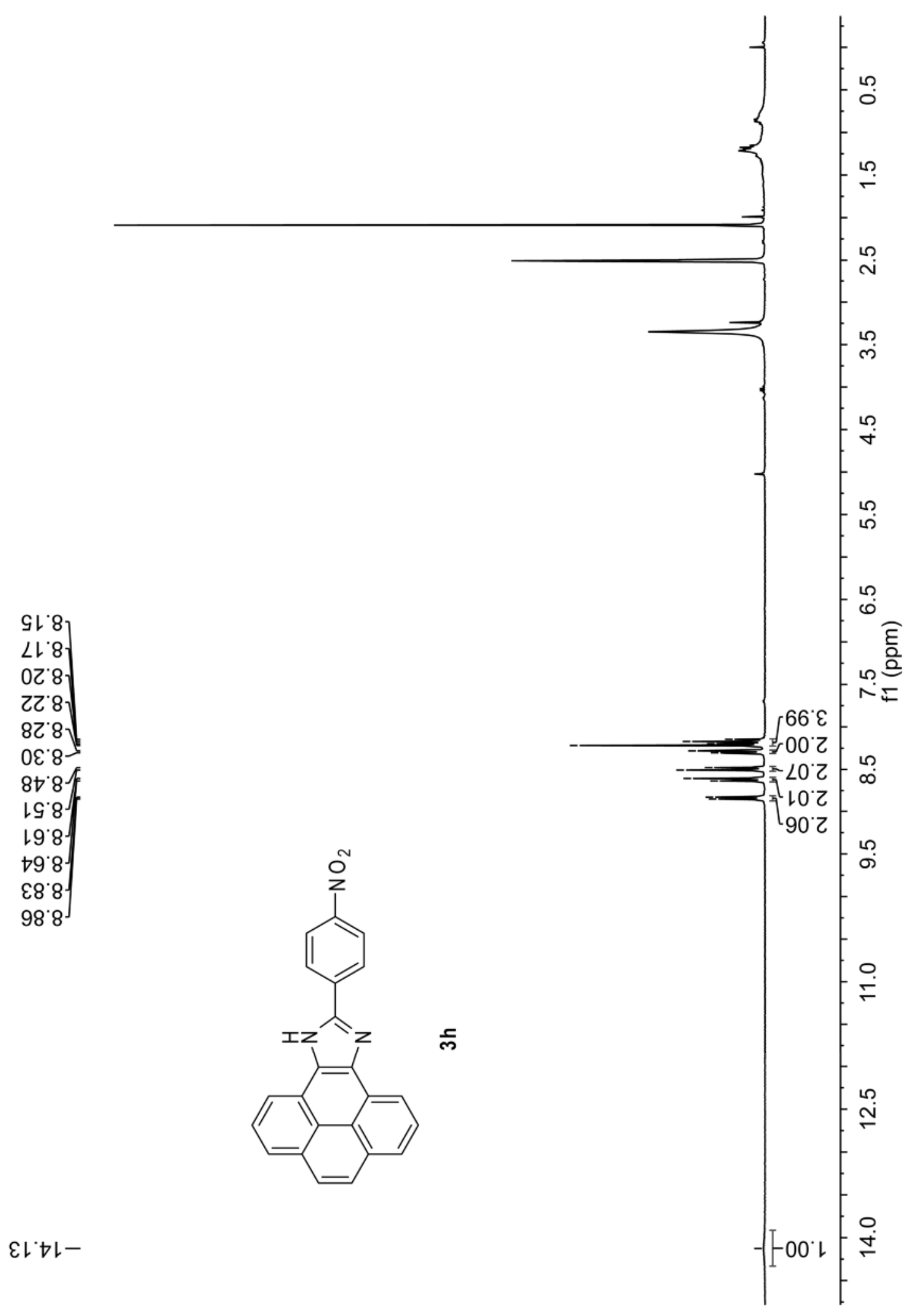

Fig. S-11 ${ }^{1} \mathrm{H}$ NMR (300 MHz, DMSO-d6) spectrum of compound $\mathbf{3 h}$. 


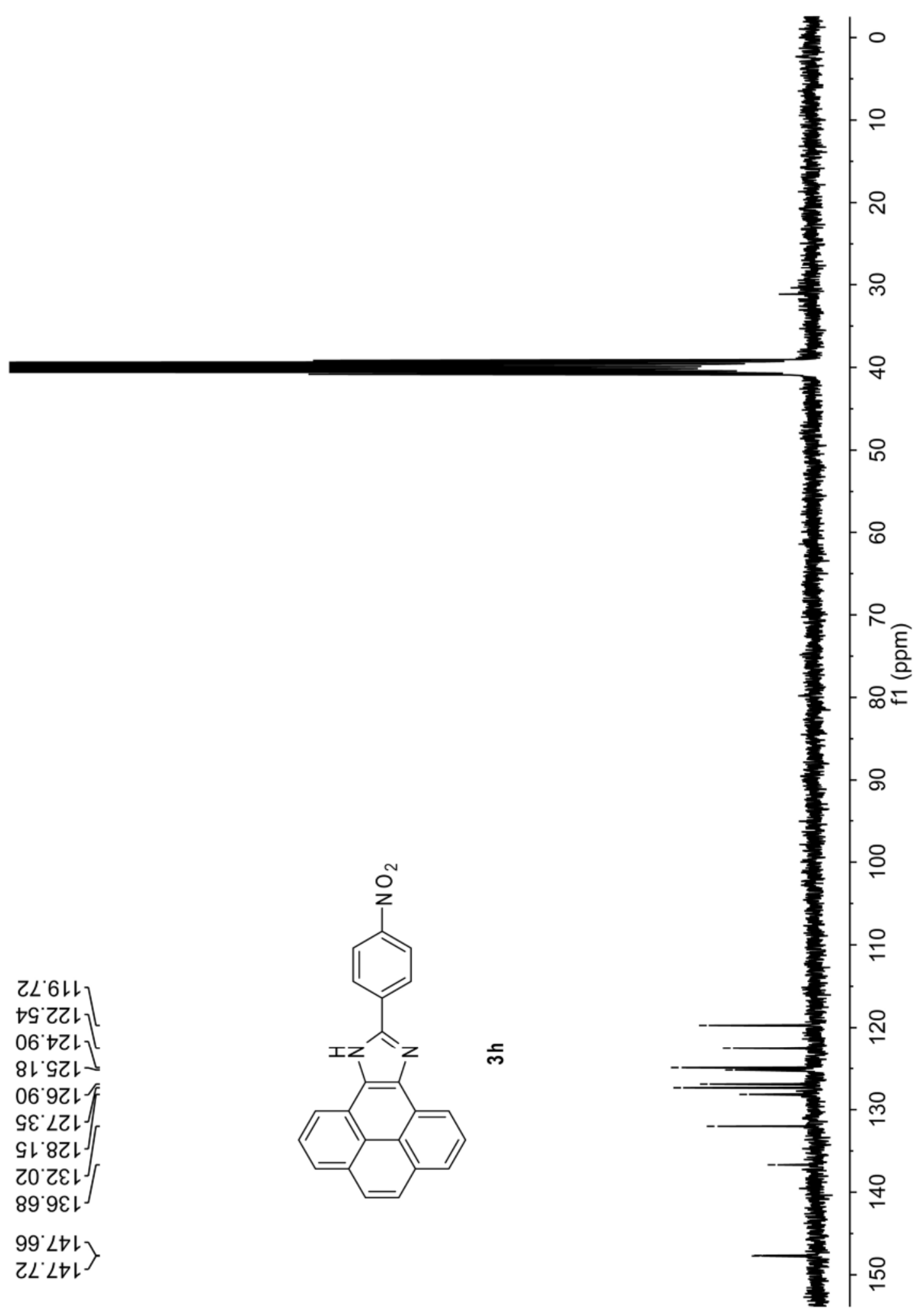

Fig. S-12 ${ }^{13} \mathrm{C}$ NMR (75 MHz, DMSO- $d_{6}$ ) spectrum of compound $\mathbf{3 h}$. 


\section{DFT and TD-DFT Computational Results}

\subsection{Geometry Optimization}

Molecular geometries of compounds 3a-h were optimized at the M06-2X/Def2-SV(P) level using Spartan'18 Parallel Suite (Wavefunction Inc.). Detailed Cartesian coordinates are provided below.

Table S-1 Cartesian coordinates of optimized structures of $\mathbf{3 a - h}$

\begin{tabular}{|c|c|c|c|c|c|c|c|c|c|c|c|}
\hline \multicolumn{4}{|l|}{$3 \mathbf{a}$} & \multicolumn{4}{|c|}{$[\mathbf{3 b}-\mathrm{H}]^{-}$anion } & \multicolumn{4}{|c|}{$3 \mathbf{b}$} \\
\hline $\mathrm{H}$ & -4.626273 & -0.046530 & 2.436749 & $\mathrm{H}$ & 4.630607 & -0.002574 & 2.347412 & $\mathrm{H}$ & 4.647183 & 0.055163 & 3.040838 \\
\hline $\mathrm{C}$ & -3.534060 & -0.035208 & 2.418490 & $\mathrm{C}$ & 3.536489 & -0.002242 & 2.336692 & $\mathrm{C}$ & 3.555004 & 0.041711 & 3.023115 \\
\hline $\mathrm{C}$ & -0.715632 & -0.005918 & 2.369580 & $\mathrm{C}$ & 0.720591 & -0.000529 & 2.307520 & $\mathrm{C}$ & 0.736331 & 0.007204 & 2.975367 \\
\hline $\mathrm{C}$ & -2.863544 & -0.024052 & 1.195123 & $\mathrm{C}$ & 2.857281 & -0.002302 & 1.118985 & $\mathrm{C}$ & 2.883713 & 0.028034 & 1.800113 \\
\hline $\mathrm{C}$ & -2.820029 & -0.031866 & 3.610291 & $\mathrm{C}$ & 2.834752 & -0.001565 & 3.539277 & C & 2.841548 & 0.038137 & 4.215230 \\
\hline $\mathrm{C}$ & -1.415172 & -0.016753 & 3.607736 & $\mathrm{C}$ & 1.429643 & -0.000842 & 3.541623 & $\mathrm{C}$ & 1.436511 & 0.020384 & 4.213246 \\
\hline $\mathrm{C}$ & -1.466754 & -0.010142 & 1.153666 & $\mathrm{C}$ & 1.457349 & -0.001452 & 1.079591 & $\mathrm{C}$ & 1.486608 & 0.011481 & 1.759039 \\
\hline $\mathrm{H}$ & -3.438736 & -0.025554 & 0.265121 & $\mathrm{H}$ & 3.392668 & -0.002803 & 0.166712 & $\mathrm{H}$ & 3.458347 & 0.029806 & 0.869773 \\
\hline $\mathrm{H}$ & -3.347444 & -0.040802 & 4.567710 & $\mathrm{H}$ & 3.367715 & -0.001536 & 4.494980 & $\mathrm{H}$ & 3.369395 & 0.049002 & 5.172363 \\
\hline $\mathrm{C}$ & 0.725553 & 0.009924 & 2.362400 & $\mathrm{C}$ & -0.720591 & 0.000529 & 2.307520 & $\mathrm{C}$ & -0.704525 & -0.010918 & 2.968592 \\
\hline $\mathrm{C}$ & 3.540754 & 0.042027 & 2.378308 & $\mathrm{C}$ & -3.536489 & 0.002242 & 2.336692 & $\mathrm{C}$ & -3.519900 & -0.047687 & 2.985440 \\
\hline $\mathrm{C}$ & 1.462087 & 0.020232 & 1.142282 & $\mathrm{C}$ & -1.457349 & 0.001452 & 1.079591 & $\mathrm{C}$ & -1.441138 & -0.023066 & 1.748525 \\
\hline $\mathrm{C}$ & 1.436894 & 0.015262 & 3.594251 & $\mathrm{C}$ & -1.429643 & 0.000842 & 3.541623 & $\mathrm{C}$ & -1.415591 & -0.016666 & 4.200710 \\
\hline $\mathrm{C}$ & 2.841058 & 0.031517 & 3.579697 & $\mathrm{C}$ & -2.834752 & 0.001565 & 3.539277 & $\mathrm{C}$ & -2.819833 & -0.035321 & 4.186624 \\
\hline $\mathrm{C}$ & 2.856940 & 0.036328 & 1.161877 & $\mathrm{C}$ & -2.857281 & 0.002302 & 1.118985 & C & -2.836246 & -0.041479 & 1.768842 \\
\hline $\mathrm{H}$ & 3.379573 & 0.035969 & 4.531182 & $\mathrm{H}$ & -3.367715 & 0.001536 & 4.494980 & $\mathrm{H}$ & -3.358055 & -0.040226 & 5.138248 \\
\hline $\mathrm{H}$ & 3.392027 & 0.044824 & 0.210064 & $\mathrm{H}$ & -3.392668 & 0.002803 & 0.166712 & $\mathrm{H}$ & -3.371459 & -0.051521 & 0.817103 \\
\hline $\mathrm{H}$ & 4.633276 & 0.055097 & 2.386273 & $\mathrm{H}$ & -4.630607 & 0.002574 & 2.347412 & $\mathrm{H}$ & -4.612379 & -0.062615 & 2.993718 \\
\hline $\mathrm{C}$ & -0.661519 & -0.011554 & 4.836881 & $\mathrm{C}$ & 0.678701 & -0.000471 & 4.773018 & $\mathrm{C}$ & 0.683233 & 0.014842 & 5.442551 \\
\hline $\mathrm{H}$ & -1.214167 & -0.020321 & 5.780153 & $\mathrm{H}$ & 1.235732 & -0.000674 & 5.715425 & $\mathrm{H}$ & 1.236050 & 0.025283 & 6.385686 \\
\hline $\mathrm{C}$ & 0.694899 & 0.003549 & 4.829809 & $\mathrm{C}$ & -0.678701 & 0.000471 & 4.773018 & $\mathrm{C}$ & -0.673179 & -0.002984 & 5.435952 \\
\hline $\mathrm{H}$ & 1.255911 & 0.007558 & 5.768350 & $\mathrm{H}$ & -1.235732 & 0.000674 & 5.715425 & $\mathrm{H}$ & -1.233744 & -0.007386 & 6.374741 \\
\hline $\mathrm{C}$ & -0.689553 & 0.000403 & -0.053504 & $\mathrm{C}$ & 0.706309 & -0.001147 & -0.146304 & $\mathrm{C}$ & 0.709429 & -0.001503 & 0.552285 \\
\hline $\mathrm{C}$ & 0.697531 & 0.012520 & -0.080569 & $\mathrm{C}$ & -0.706309 & 0.001147 & -0.146304 & $\mathrm{C}$ & -0.677503 & -0.015108 & 0.525347 \\
\hline $\mathrm{N}$ & -1.066663 & -0.000697 & -1.373036 & $\mathrm{~N}$ & 1.146587 & -0.002073 & -1.420336 & $\mathrm{~N}$ & 1.086260 & -0.000967 & -0.767543 \\
\hline $\mathrm{H}$ & -2.013060 & -0.039804 & -1.726259 & $\mathrm{~N}$ & -1.146587 & 0.002073 & -1.420336 & $\mathrm{H}$ & 2.032221 & 0.040192 & -1.121634 \\
\hline $\mathrm{N}$ & 1.154976 & 0.015633 & -1.366157 & $\mathrm{C}$ & 0.000000 & 0.000000 & -2.129728 & $\mathrm{~N}$ & -1.135680 & -0.019393 & -0.759965 \\
\hline $\mathrm{C}$ & 0.082380 & 0.007495 & -2.124976 & $\mathrm{C}$ & 0.000000 & 0.000000 & -3.600669 & $\mathrm{C}$ & -0.063181 & -0.010581 & -1.519123 \\
\hline $\mathrm{C}$ & 0.091036 & 0.003874 & -3.594276 & $\mathrm{C}$ & -0.000000 & 0.000000 & -6.416655 & $\mathrm{C}$ & -0.070719 & -0.007170 & -2.987671 \\
\hline $\mathrm{C}$ & 0.205733 & -0.014125 & -6.394255 & $\mathrm{C}$ & 1.206839 & -0.012377 & -4.317405 & $\mathrm{C}$ & -0.182711 & 0.019038 & -5.812230 \\
\hline $\mathrm{C}$ & -1.077027 & 0.145225 & -4.354553 & $\mathrm{C}$ & -1.206839 & 0.012377 & -4.317405 & $\mathrm{C}$ & 1.092725 & -0.159014 & -3.750420 \\
\hline $\mathrm{C}$ & 1.319817 & -0.142314 & -4.253891 & $\mathrm{C}$ & -1.205166 & 0.012724 & -5.710048 & C & -1.293644 & 0.154471 & -3.652443 \\
\hline $\mathrm{C}$ & 1.374685 & -0.150931 & -5.643020 & $\mathrm{C}$ & 1.205166 & -0.012724 & -5.710048 & $\mathrm{C}$ & -1.342973 & 0.167982 & -5.040865 \\
\hline $\mathrm{C}$ & -1.019498 & 0.135080 & -5.746385 & $\mathrm{H}$ & 2.137032 & -0.022384 & -3.744756 & $\mathrm{C}$ & 1.033880 & -0.142855 & -5.141684 \\
\hline $\mathrm{H}$ & -2.048360 & 0.281025 & -3.870185 & $\mathrm{H}$ & -2.137031 & 0.022384 & -3.744756 & $\mathrm{H}$ & 2.064230 & -0.303925 & -3.268820 \\
\hline $\mathrm{H}$ & 2.222923 & -0.247393 & -3.649595 & $\mathrm{H}$ & -2.154973 & 0.024302 & -6.253619 & $\mathrm{H}$ & -2.198211 & 0.272809 & -3.052646 \\
\hline $\mathrm{H}$ & 2.338035 & -0.268326 & -6.144530 & $\mathrm{H}$ & 2.154973 & -0.024302 & -6.253619 & $\mathrm{H}$ & -2.305748 & 0.302407 & -5.542430 \\
\hline $\mathrm{H}$ & -1.938897 & 0.248772 & -6.325112 & $\mathrm{H}$ & -0.000000 & 0.000000 & -7.510481 & $\mathrm{H}$ & 1.955431 & -0.261578 & -5.718669 \\
\hline $\mathrm{H}$ & 0.250301 & -0.020022 & -7.485688 & & & & & $\mathrm{C}$ & -0.248581 & 0.003697 & -7.317145 \\
\hline & & & & & & & & $\mathrm{H}$ & 0.696869 & 0.350866 & -7.762803 \\
\hline & & & & & & & & $\mathrm{H}$ & -1.062411 & 0.647499 & -7.686262 \\
\hline & & & & & & & & $\mathrm{H}$ & -0.437560 & -1.018042 & -7.691095 \\
\hline $3 c$ & & & & $3 \mathbf{e}$ & & & & 3f & & & \\
\hline $\mathrm{H}$ & -4.627827 & -0.028604 & 2.460383 & $\mathrm{H}$ & 4.812222 & 0.000000 & 3.190593 & $\mathrm{H}$ & -4.754355 & -0.051809 & 2.839716 \\
\hline $\mathrm{C}$ & -3.535646 & -0.021468 & 2.440354 & $\mathrm{C}$ & 3.719773 & 0.000000 & 3.184990 & $\mathrm{C}$ & -3.662636 & -0.039121 & 2.803620 \\
\hline $\mathrm{C}$ & -0.717002 & -0.003021 & 2.387041 & $\mathrm{C}$ & 0.900987 & 0.000000 & 3.168672 & C & -0.844985 & -0.006638 & 2.708926 \\
\hline $\mathrm{C}$ & -2.866949 & -0.011725 & 1.215925 & $\mathrm{C}$ & 3.035202 & 0.000000 & 1.969469 & $\mathrm{C}$ & -3.011919 & -0.030597 & 1.569463 \\
\hline $\mathrm{C}$ & -2.819564 & -0.022095 & 3.630864 & $\mathrm{C}$ & 3.019763 & 0.000000 & 4.385156 & $\mathrm{C}$ & -2.928994 & -0.031674 & 3.983396 \\
\hline $\mathrm{C}$ & -1.414612 & -0.012481 & 3.626272 & $\mathrm{C}$ & 1.614848 & 0.000000 & 4.398634 & C & -1.524224 & -0.015267 & 3.958290 \\
\hline $\mathrm{C}$ & -1.470268 & -0.003152 & 1.172543 & $\mathrm{C}$ & 1.637689 & 0.000000 & 1.943766 & $\mathrm{C}$ & -1.616168 & -0.014652 & 1.505646 \\
\hline $\mathrm{H}$ & -3.443813 & -0.010285 & 0.286940 & $\mathrm{H}$ & 3.599824 & 0.000000 & 1.033007 & $\mathrm{H}$ & -3.602272 & -0.036526 & 0.648969 \\
\hline $\mathrm{H}$ & -3.345488 & -0.029876 & 4.589092 & $\mathrm{H}$ & 3.558242 & 0.000000 & 5.336470 & $\mathrm{H}$ & -3.440659 & -0.038601 & 4.949317 \\
\hline $\mathrm{C}$ & 0.724258 & 0.007213 & 2.377798 & $\mathrm{C}$ & -0.539905 & 0.000000 & 3.177458 & $\mathrm{C}$ & 0.596038 & 0.010043 & 2.679084 \\
\hline $\mathrm{C}$ & 3.539710 & 0.027568 & 2.389797 & $\mathrm{C}$ & -3.354876 & 0.000000 & 3.224990 & $\mathrm{C}$ & 3.411329 & 0.042796 & 2.649780 \\
\hline $\mathrm{C}$ & 1.459259 & 0.016180 & 1.156854 & $\mathrm{C}$ & -1.289896 & 0.000000 & 1.965217 & $\mathrm{C}$ & 1.313366 & 0.018706 & 1.448017 \\
\hline
\end{tabular}




\begin{tabular}{|c|c|c|c|c|c|c|c|c|c|c|c|}
\hline $\mathrm{C}$ & 1.437304 & 0.008061 & 3.608615 & $\mathrm{C}$ & -1.237457 & 0.000000 & 4.417328 & $\mathrm{C}$ & 1.327001 & 0.017978 & 3.899300 \\
\hline $\mathrm{C}$ & 2.841571 & 0.018447 & 3.591990 & $\mathrm{C}$ & -2.641700 & 0.000000 & 4.418706 & $\mathrm{C}$ & 2.730853 & 0.034460 & 3.861941 \\
\hline $\mathrm{C}$ & 2.854101 & 0.026355 & 1.174294 & $\mathrm{C}$ & -2.684753 & 0.000000 & 2.001006 & $\mathrm{C}$ & 2.708113 & 0.034935 & 1.444236 \\
\hline $\mathrm{H}$ & 3.381434 & 0.019304 & 4.542694 & $\mathrm{H}$ & -3.169478 & 0.000000 & 5.376216 & $\mathrm{H}$ & 3.284516 & 0.040729 & 4.804678 \\
\hline $\mathrm{H}$ & 3.388291 & 0.033605 & 0.221975 & $\mathrm{H}$ & -3.230641 & 0.000000 & 1.055258 & $\mathrm{H}$ & 3.228361 & 0.041463 & 0.484197 \\
\hline $\mathrm{H}$ & 4.632253 & 0.035933 & 2.396263 & $\mathrm{H}$ & -4.447373 & 0.000000 & 3.245112 & $\mathrm{H}$ & 4.503758 & 0.055701 & 2.640050 \\
\hline $\mathrm{C}$ & -0.659262 & -0.011524 & 4.854350 & $\mathrm{C}$ & 0.875027 & 0.000000 & 5.636307 & $\mathrm{C}$ & -0.750976 & -0.006924 & 5.175254 \\
\hline $\mathrm{H}$ & -1.210586 & -0.019172 & 5.798368 & $\mathrm{H}$ & 1.438501 & 0.000000 & 6.573240 & $\mathrm{H}$ & -1.288544 & -0.013891 & 6.127168 \\
\hline $\mathrm{C}$ & 0.697194 & -0.002107 & 4.845276 & $\mathrm{C}$ & -0.481449 & 0.000000 & 5.644469 & $\mathrm{C}$ & 0.605167 & 0.008832 & 5.146541 \\
\hline $\mathrm{H}$ & 1.259632 & -0.001514 & 5.782941 & $\mathrm{H}$ & -1.032128 & 0.000000 & 6.589142 & $\mathrm{H}$ & 1.181024 & 0.014962 & 6.075993 \\
\hline $\mathrm{C}$ & -0.694588 & 0.005504 & -0.035760 & $\mathrm{C}$ & 0.847112 & 0.000000 & 0.746211 & $\mathrm{C}$ & -0.857893 & -0.005434 & 0.285984 \\
\hline $\mathrm{C}$ & 0.692484 & 0.012823 & -0.064714 & $\mathrm{C}$ & -0.539616 & 0.000000 & 0.733779 & $\mathrm{C}$ & 0.528872 & 0.009787 & 0.237865 \\
\hline $\mathrm{N}$ & -1.074204 & 0.006553 & -1.354814 & $\mathrm{~N}$ & 1.209824 & 0.000000 & -0.578228 & $\mathrm{~N}$ & -1.255537 & -0.009425 & -1.027572 \\
\hline $\mathrm{H}$ & -2.022171 & -0.025218 & -1.704504 & $\mathrm{H}$ & 2.153543 & 0.000000 & -0.939950 & $\mathrm{H}$ & -2.209062 & -0.021793 & -1.362672 \\
\hline $\mathrm{N}$ & 1.147665 & 0.015596 & -1.351019 & $\mathrm{~N}$ & -1.011202 & 0.000000 & -0.547253 & $\mathrm{~N}$ & 0.966073 & 0.014321 & -1.053897 \\
\hline $\mathrm{C}$ & 0.073909 & 0.011325 & -2.107916 & $\mathrm{C}$ & 0.053097 & 0.000000 & -1.318115 & $\mathrm{C}$ & -0.117160 & 0.002127 & -1.795815 \\
\hline $\mathrm{C}$ & 0.085450 & 0.007171 & -3.576095 & $\mathrm{C}$ & 0.028981 & 0.000000 & -2.785175 & $\mathrm{C}$ & -0.120681 & 0.000690 & -3.265076 \\
\hline $\mathrm{C}$ & 0.212763 & -0.017037 & -6.365643 & $\mathrm{C}$ & -0.125238 & 0.000000 & -5.595664 & $\mathrm{C}$ & -0.037526 & -0.002372 & -6.069867 \\
\hline $\mathrm{C}$ & -1.080768 & 0.114531 & -4.343396 & $\mathrm{C}$ & 1.193869 & 0.000000 & -3.568825 & $\mathrm{C}$ & 1.107747 & -0.001780 & -3.932346 \\
\hline $\mathrm{C}$ & 1.319451 & -0.106919 & -4.232192 & $\mathrm{C}$ & -1.208251 & 0.000000 & -3.435360 & $\mathrm{C}$ & -1.304676 & 0.002160 & -4.019229 \\
\hline $\mathrm{C}$ & 1.387581 & -0.118953 & -5.619530 & $\mathrm{C}$ & -1.295042 & 0.000000 & -4.824837 & $\mathrm{C}$ & -1.265032 & 0.000432 & -5.411847 \\
\hline $\mathrm{C}$ & -1.023538 & 0.101306 & -5.734649 & $\mathrm{C}$ & 1.121306 & 0.000000 & -4.952833 & $\mathrm{C}$ & 1.146673 & -0.003352 & -5.326528 \\
\hline $\mathrm{H}$ & -2.059279 & 0.223222 & -3.867576 & $\mathrm{H}$ & 2.183753 & 0.000000 & -3.103224 & $\mathrm{H}$ & 2.037577 & -0.002631 & -3.358950 \\
\hline $\mathrm{H}$ & 2.224127 & -0.185616 & -3.626321 & $\mathrm{H}$ & -2.112908 & 0.000000 & -2.823877 & $\mathrm{H}$ & -2.279972 & 0.006006 & -3.524146 \\
\hline $\mathrm{H}$ & 2.346399 & -0.209688 & -6.132752 & $\mathrm{H}$ & -2.279166 & 0.000000 & -5.294368 & $\mathrm{H}$ & -2.196505 & 0.001874 & -5.981866 \\
\hline $\mathrm{H}$ & -1.931969 & 0.185708 & -6.333350 & $\mathrm{H}$ & 2.020748 & 0.000000 & -5.571120 & $\mathrm{H}$ & 0.006613 & -0.003501 & -7.163178 \\
\hline $\mathrm{Cl}$ & 0.292700 & -0.035948 & -8.100397 & $\mathrm{O}$ & -0.102024 & 0.000000 & -6.944191 & $\mathrm{C}$ & 2.462450 & -0.006382 & -6.025402 \\
\hline & & & & $\mathrm{C}$ & -1.324222 & 0.000000 & -7.633606 & $\mathrm{H}$ & 2.399726 & -0.009616 & -7.142602 \\
\hline & & & & $\mathrm{H}$ & -1.919584 & -0.900440 & -7.397377 & $\mathrm{O}$ & 3.524520 & -0.006016 & -5.466439 \\
\hline & & & & $\mathrm{H}$ & -1.077821 & 0.000000 & -8.703819 & & & & \\
\hline & & & & $\mathrm{H}$ & -1.919584 & 0.900440 & -7.397377 & & & & \\
\hline $3 g$ & & & & $3 \mathrm{~h}$ & & & & & & & \\
\hline $\mathrm{H}$ & 4.639477 & 0.000000 & 2.863502 & $\mathrm{H}$ & 4.640295 & 0.000000 & 2.873281 & & & & \\
\hline $\mathrm{C}$ & 3.547341 & 0.000000 & 2.843444 & $\mathrm{C}$ & 3.548228 & 0.000000 & 2.849664 & & & & \\
\hline $\mathrm{C}$ & 0.728286 & 0.000000 & 2.790329 & $\mathrm{C}$ & 0.729296 & 0.000000 & 2.787673 & & & & \\
\hline $\mathrm{C}$ & 2.878434 & 0.000000 & 1.619104 & $\mathrm{C}$ & 2.883079 & 0.000000 & 1.623181 & & & & \\
\hline $\mathrm{C}$ & 2.831021 & 0.000000 & 4.033622 & $\mathrm{C}$ & 2.828082 & 0.000000 & 4.037279 & & & & \\
\hline $\mathrm{C}$ & 1.425875 & 0.000000 & 4.029438 & $\mathrm{C}$ & 1.423021 & 0.000000 & 4.028833 & & & & \\
\hline $\mathrm{C}$ & 1.481971 & 0.000000 & 1.576424 & $\mathrm{C}$ & 1.486992 & 0.000000 & 1.576466 & & & & \\
\hline $\mathrm{H}$ & 3.455294 & 0.000000 & 0.690141 & $\mathrm{H}$ & 3.463033 & 0.000000 & 0.696059 & & & & \\
\hline $\mathrm{H}$ & 3.357033 & 0.000000 & 4.991817 & $\mathrm{H}$ & 3.351136 & 0.000000 & 4.997115 & & & & \\
\hline $\mathrm{C}$ & -0.713269 & 0.000000 & 2.781157 & $\mathrm{C}$ & -0.712327 & 0.000000 & 2.774694 & & & & \\
\hline $\mathrm{C}$ & -3.528759 & 0.000000 & 2.794186 & $\mathrm{C}$ & -3.527771 & 0.000000 & 2.780138 & & & & \\
\hline $\mathrm{C}$ & -1.448905 & 0.000000 & 1.560643 & $\mathrm{C}$ & -1.444835 & 0.000000 & 1.552777 & & & & \\
\hline $\mathrm{C}$ & -1.425677 & 0.000000 & 4.012136 & $\mathrm{C}$ & -1.427993 & 0.000000 & 4.003579 & & & & \\
\hline $\mathrm{C}$ & -2.830156 & 0.000000 & 3.995866 & $\mathrm{C}$ & -2.832399 & 0.000000 & 3.983450 & & & & \\
\hline $\mathrm{C}$ & -2.843500 & 0.000000 & 1.578371 & $\mathrm{C}$ & -2.839208 & 0.000000 & 1.566135 & & & & \\
\hline $\mathrm{H}$ & -3.369651 & 0.000000 & 4.946754 & $\mathrm{H}$ & -3.374594 & 0.000000 & 4.932823 & & & & \\
\hline $\mathrm{H}$ & -3.378433 & 0.000000 & 0.626468 & $\mathrm{H}$ & -3.372004 & 0.000000 & 0.612991 & & & & \\
\hline $\mathrm{H}$ & -4.621283 & 0.000000 & 2.800987 & $\mathrm{H}$ & -4.620311 & 0.000000 & 2.783898 & & & & \\
\hline $\mathrm{C}$ & 0.670885 & 0.000000 & 5.257684 & $\mathrm{C}$ & 0.664794 & 0.000000 & 5.254876 & & & & \\
\hline $\mathrm{H}$ & 1.222368 & 0.000000 & 6.201589 & $\mathrm{H}$ & 1.213463 & 0.000000 & 6.200428 & & & & \\
\hline $\mathrm{C}$ & -0.685473 & 0.000000 & 5.248790 & $\mathrm{C}$ & -0.691501 & 0.000000 & 5.242148 & & & & \\
\hline $\mathrm{H}$ & -1.247728 & 0.000000 & 6.186501 & $\mathrm{H}$ & -1.256333 & 0.000000 & 6.178326 & & & & \\
\hline $\mathrm{C}$ & 0.706195 & 0.000000 & 0.367726 & $\mathrm{C}$ & 0.714482 & 0.000000 & 0.365379 & & & & \\
\hline $\mathrm{C}$ & -0.681998 & 0.000000 & 0.338672 & $\mathrm{C}$ & -0.673992 & 0.000000 & 0.333211 & & & & \\
\hline $\mathrm{N}$ & 1.086256 & 0.000000 & -0.949391 & $\mathrm{~N}$ & 1.097966 & 0.000000 & -0.950846 & & & & \\
\hline $\mathrm{H}$ & 2.035475 & 0.000000 & -1.297404 & $\mathrm{H}$ & 2.048527 & 0.000000 & -1.295327 & & & & \\
\hline $\mathrm{N}$ & -1.135780 & 0.000000 & -0.946594 & $\mathrm{~N}$ & -1.124096 & 0.000000 & -0.953176 & & & & \\
\hline $\mathrm{C}$ & -0.061306 & 0.000000 & -1.703807 & $\mathrm{C}$ & -0.048023 & 0.000000 & -1.707624 & & & & \\
\hline $\mathrm{C}$ & -0.072385 & 0.000000 & -3.171291 & $\mathrm{C}$ & -0.062295 & 0.000000 & -3.174422 & & & & \\
\hline $\mathrm{C}$ & -0.190361 & 0.000000 & -5.966538 & $\mathrm{C}$ & -0.197108 & 0.000000 & -5.941385 & & & & \\
\hline $\mathrm{C}$ & 1.108319 & 0.000000 & -3.932884 & $\mathrm{C}$ & 1.111955 & 0.000000 & -3.941691 & & & & \\
\hline $\mathrm{C}$ & -1.313357 & 0.000000 & -3.824947 & $\mathrm{C}$ & -1.307599 & 0.000000 & -3.823362 & & & & \\
\hline $\mathrm{C}$ & -1.368146 & 0.000000 & -5.212854 & $\mathrm{C}$ & -1.381005 & 0.000000 & -5.208884 & & & & \\
\hline $\mathrm{C}$ & 1.049800 & 0.000000 & -5.319236 & $\mathrm{C}$ & 1.050969 & 0.000000 & -5.330912 & & & & \\
\hline $\mathrm{H}$ & 2.087935 & 0.000000 & -3.447096 & $\mathrm{H}$ & 2.095701 & 0.000000 & -3.465643 & & & & \\
\hline $\mathrm{H}$ & -2.219310 & 0.000000 & -3.216463 & $\mathrm{H}$ & -2.211407 & 0.000000 & -3.211988 & & & & \\
\hline $\mathrm{H}$ & -2.336129 & 0.000000 & -5.723389 & $\mathrm{H}$ & -2.336778 & 0.000000 & -5.732689 & & & & \\
\hline $\mathrm{H}$ & 1.957052 & 0.000000 & -5.928082 & $\mathrm{H}$ & 1.950679 & 0.000000 & -5.946017 & & & & \\
\hline
\end{tabular}




\begin{tabular}{|lrll|lrrr|l|}
\hline $\mathrm{C}$ & -0.245211 & 0.000000 & -7.450611 & $\mathrm{~N}$ & -0.268603 & 0.000000 & -7.414613 & \\
$\mathrm{H}$ & -1.276387 & 0.000000 & -7.883540 & $\mathrm{O}$ & 0.774881 & 0.000000 & -8.021386 & \\
$\mathrm{O}$ & 0.724189 & 0.000000 & -8.161222 & $\mathrm{O}$ & -1.366397 & 0.000000 & -7.914437 & \\
\hline
\end{tabular}

\subsection{Results of QTAIM Analysis}

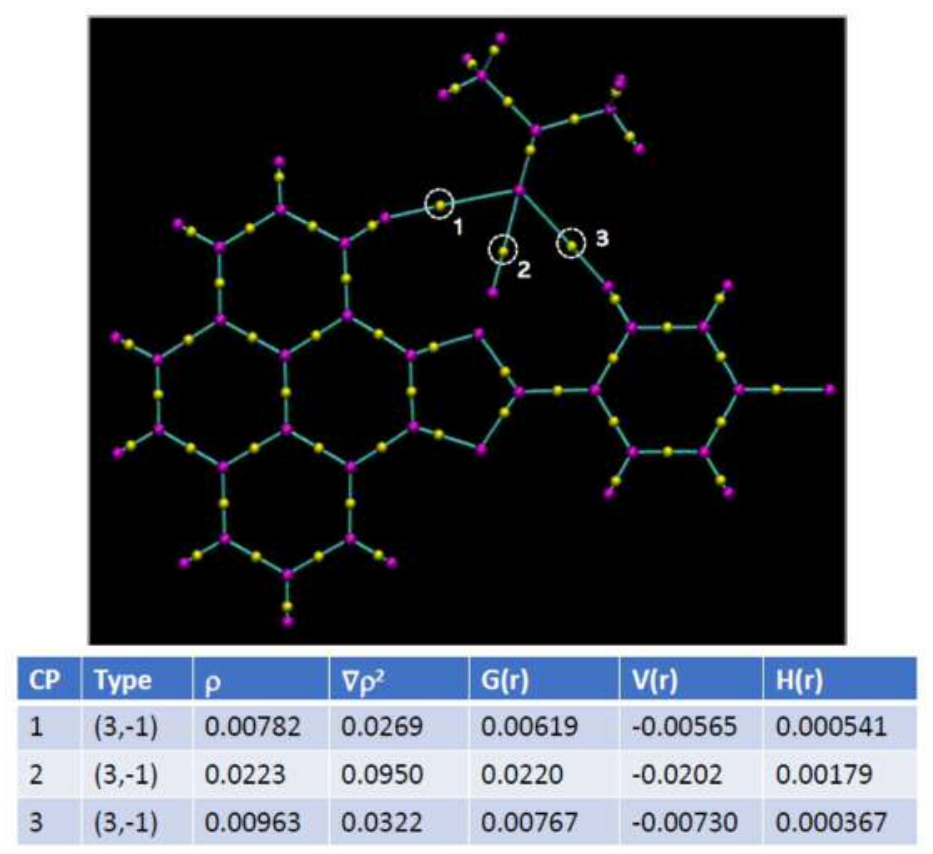

Fig. S-13 Molecular graph of compound 3c complexed with acetone. Bond critical points (BCPs) for hydrogen bonding interactions are highlighted. Calculated at the M06-2X/Def2-SV(P) level.

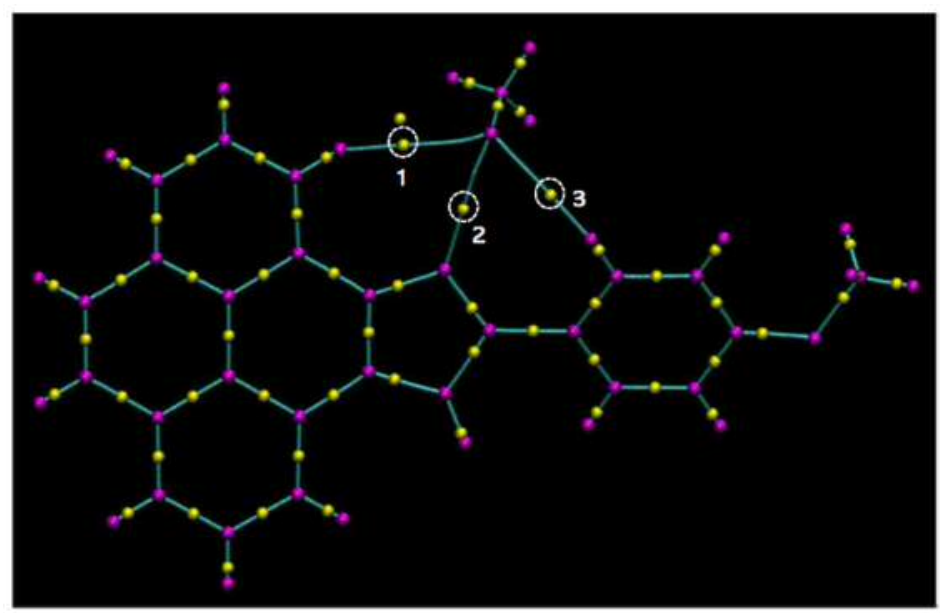

\begin{tabular}{|l|l|l|l|l|l|l|}
\hline CP & Type & $\rho$ & $\nabla \rho^{2}$ & $\mathrm{G}(\mathrm{r})$ & $\mathrm{V}(\mathrm{r})$ & $\mathrm{H}(\mathrm{r})$ \\
\hline 1 & $(3,-1)$ & 0.0311 & 0.109 & 0.0268 & -0.0264 & 0.000391 \\
\hline 2 & $(3,-1)$ & 0.00909 & 0.0316 & 0.00746 & -0.00703 & 0.000433 \\
\hline 3 & $(3,-1)$ & 0.00625 & 0.0257 & 0.00558 & -0.00473 & 0.000845 \\
\hline
\end{tabular}

Fig. S-14 Molecular graph of compound 3e complexed with methanol. Bond critical points (BCPs) for hydrogen bonding interactions are highlighted. Calculated at the M06-2X/Def2$\mathrm{SV}(\mathrm{P})$ level. 


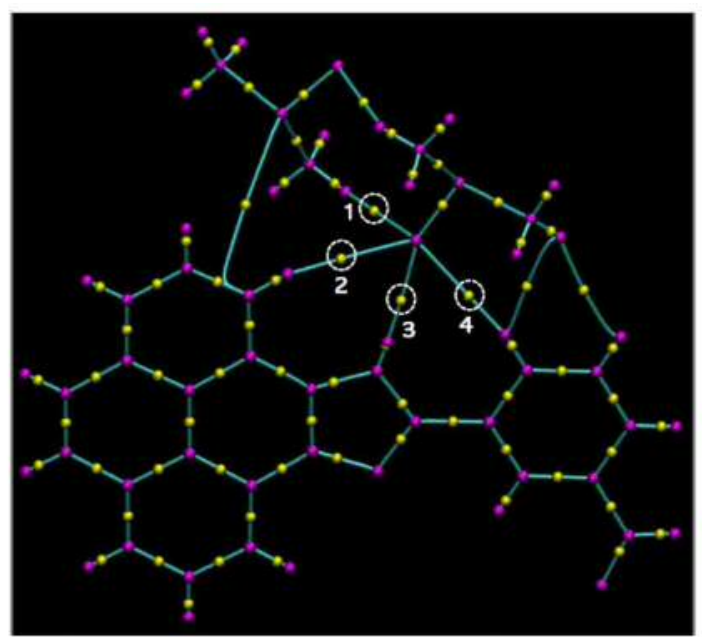

\begin{tabular}{|l|l|l|l|l|l|l|}
\hline CP & Type & $\rho$ & $\nabla \rho^{2}$ & $G(r)$ & $V(r)$ & $H(r)$ \\
\hline 1 & $(3,-1)$ & 0.0111 & 0.0378 & 0.00920 & -0.00896 & 0.000242 \\
\hline 2 & $(3,-1)$ & 0.0719 & 0.0263 & 0.00594 & -0.00530 & 0.000640 \\
\hline 3 & $(3,-1)$ & 0.0165 & 0.0715 & 0.0165 & -0.0152 & 0.00133 \\
\hline 4 & $(3,-1)$ & 0.00866 & 0.0287 & 0.00679 & -0.00642 & 0.000380 \\
\hline
\end{tabular}

Fig. S-15 Molecular graph of compound 3f complexed with two molecules of DMSO. Bond critical points (BCPs) for hydrogen bonding interactions are highlighted. Calculated at the M062X/Def2-SV(P) level.

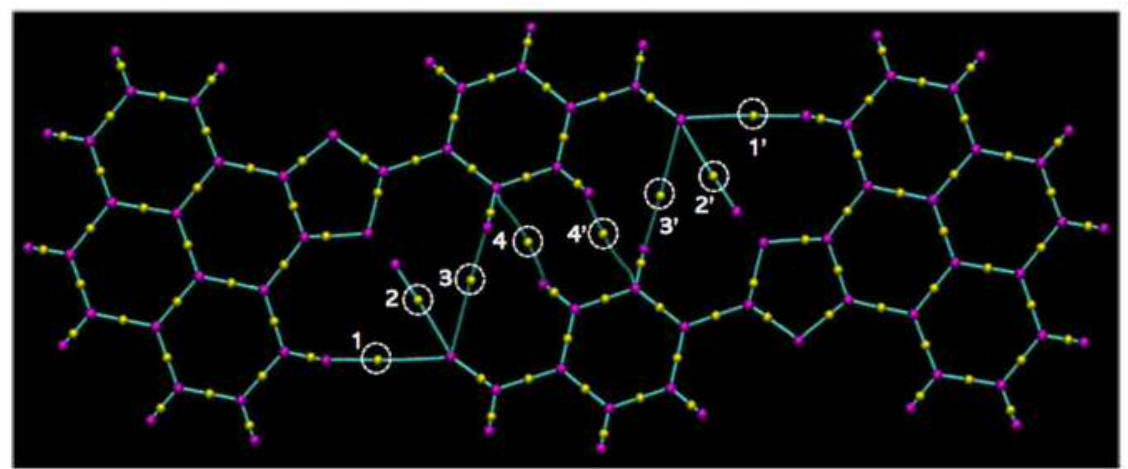

\begin{tabular}{|l|l|l|l|l|l|l|}
\hline CP & Type & $\rho$ & $\nabla \rho^{2}$ & $\mathrm{G}(\mathrm{r})$ & $\mathrm{V}(\mathrm{r})$ & $\mathrm{H}(\mathrm{r})$ \\
\hline $1 / 1^{\prime}$ & $(3,-1)$ & 0.0622 & 0.0209 & 0.00475 & -0.00428 & 0.000467 \\
\hline $2 / 2^{\prime}$ & $(3,-1)$ & 0.0113 & 0.0373 & 0.00886 & -0.00840 & 0.000464 \\
\hline $3 / 3^{\prime}$ & $(3,-1)$ & 0.00307 & 0.0133 & 0.00249 & -0.00166 & 0.000826 \\
\hline $4 / 4^{\prime}$ & $(3,-1)$ & 0.00762 & 0.0306 & 0.00582 & -0.00399 & 0.00184 \\
\hline
\end{tabular}

Fig. S-16 Molecular graph of hydrogen bonded dimer of 3g. Bond critical points (BCPs) for hydrogen bonding interactions are highlighted. Calculated at the M06-2X/Def2-SV(P) level. 


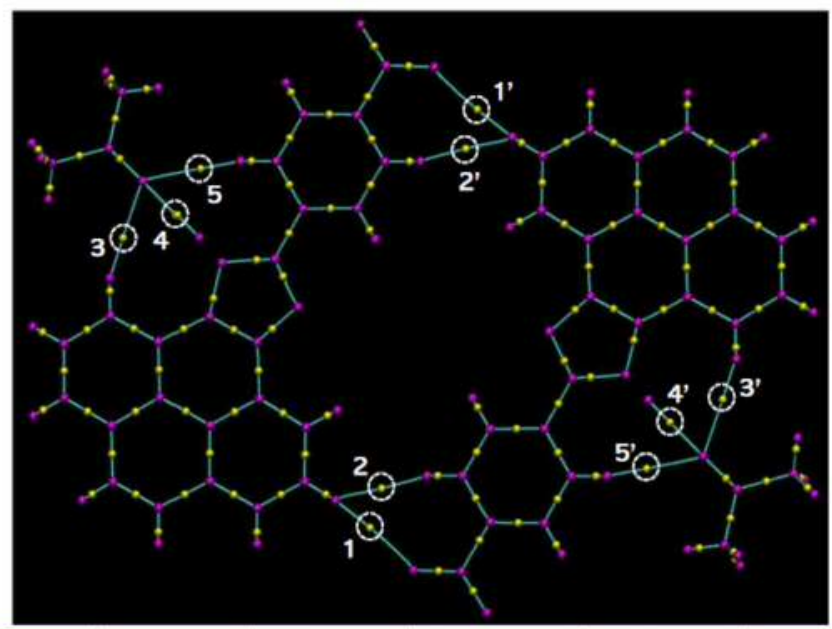

\begin{tabular}{|l|l|l|l|l|l|l|}
\hline CP & Type & $\rho$ & $\nabla \rho^{2}$ & $G(r)$ & $V(r)$ & $H(r)$ \\
\hline $1 / 1^{\prime}$ & $(3,-1)$ & 0.00612 & 0.0241 & 0.00525 & -0.00447 & 0.000782 \\
\hline $2 / 2^{\prime}$ & $(3,-1)$ & 0.00402 & 0.0193 & 0.00366 & -0.00249 & 0.00117 \\
\hline $3 / 3^{\prime}$ & $(3,-1)$ & 0.00806 & 0.0275 & 0.00637 & -0.00586 & 0.000510 \\
\hline $4 / 4^{\prime}$ & $(3,-1)$ & 0.0233 & 0.0988 & 0.0228 & -0.0210 & 0.00185 \\
\hline $5 / 5^{\prime}$ & $(3,-1)$ & 0.0102 & 0.0337 & 0.00813 & -0.00785 & 0.000286 \\
\hline
\end{tabular}

Fig. S-17 Molecular graph of hydrogen bonded dimer of $\mathbf{3 h}$. Bond critical points (BCPs) for hydrogen bonding interactions are highlighted. Calculated at the M06-2X/Def2-SV(P) level.

\subsection{Electronic Absorption Properties of Compound 3a and 3a Anion Calculated by TD-DFT}

Table S-2 Vertical electronic transitions of 3a calculated at the TD-B3LYP/6-31 1+G(d,p) level

\begin{tabular}{lll}
\hline Wavelength (nm) & Osc. Strength & Major Contributions \\
\hline 368.4 & 0.3762 & $\mathrm{HOMO} \rightarrow \mathrm{LUMO}(91 \%)$ \\
357.2 & 0.3052 & $\mathrm{HOMO} \rightarrow \mathrm{L}+1(82 \%)$ \\
328.2 & 0.1637 & $\mathrm{H}-1 \rightarrow \mathrm{LUMO}(16 \%), \mathrm{HOMO} \rightarrow \mathrm{L}+1(11 \%), \mathrm{HOMO} \rightarrow \mathrm{L}+2(65 \%)$ \\
297.8 & 0.3172 & $\mathrm{H}-2 \rightarrow \mathrm{LUMO}(28 \%), \mathrm{H}-1 \rightarrow \mathrm{LUMO}(55 \%)$ \\
289.4 & 0.1383 & $\mathrm{H}-2 \rightarrow \mathrm{LUMO}(54 \%), \mathrm{H}-1 \rightarrow \mathrm{LUMO}(11 \%), \mathrm{HOMO} \rightarrow \mathrm{L}+2(15 \%)$ \\
288.4 & 0.0319 & $\mathrm{HOMO} \rightarrow \mathrm{L}+3(83 \%)$ \\
276.0 & 0.0206 & $\mathrm{H}-2 \rightarrow \mathrm{L}+1(13 \%), \mathrm{H}-1 \rightarrow \mathrm{L}+1(38 \%), \mathrm{HOMO} \rightarrow \mathrm{L}+4(40 \%)$ \\
273.1 & 0.0896 & $\mathrm{H}-1 \rightarrow \mathrm{L}+1(44 \%), \mathrm{HOMO} \rightarrow \mathrm{L}+4(43 \%)$ \\
269.3 & 0.0852 & $\mathrm{H}-2 \rightarrow \mathrm{L}+1(67 \%)$ \\
257.3 & 0.0006 & $\mathrm{HOMO} \rightarrow \mathrm{L}+5(95 \%)$ \\
\hline
\end{tabular}


Table S-3 Vertical electronic transitions of 3a anion calculated at the TD-B3LYP/6-311+G(d,p) level

\begin{tabular}{lll}
\hline Wavelength $(\mathbf{n m})$ & Osc. Strength & Major Contributions \\
\hline 428.9 & 0.1372 & $\mathrm{HOMO} \rightarrow \mathrm{LUMO}(95 \%)$ \\
383.9 & 0.4334 & $\mathrm{HOMO} \rightarrow \mathrm{L}+1(89 \%)$ \\
346.7 & 0.3759 & $\mathrm{HOMO} \rightarrow \mathrm{L}+2(89 \%)$ \\
323.4 & 0.2509 & $\mathrm{H}-1 \rightarrow \mathrm{LUMO}(85 \%)$ \\
320.2 & 0.0054 & $\mathrm{HOMO} \rightarrow \mathrm{L}+3(96 \%)$ \\
301.2 & 0.0461 & $\mathrm{H}-2 \rightarrow \mathrm{LUMO}(74 \%), \mathrm{HOMO} \rightarrow \mathrm{L}+5(18 \%)$ \\
295.4 & 0.0016 & $\mathrm{H}-1 \rightarrow \mathrm{L}+1(43 \%), \mathrm{HOMO} \rightarrow \mathrm{L}+5(46 \%)$ \\
288.6 & 0.0002 & $\mathrm{HOMO} \rightarrow \mathrm{L}+4(93 \%)$ \\
276.9 & 0.2561 & $\mathrm{H}-1 \rightarrow \mathrm{L}+1(50 \%), \mathrm{HOMO} \rightarrow \mathrm{L}+5(28 \%)$ \\
276.1 & 0.0002 & $\mathrm{H}-3 \rightarrow \mathrm{LUMO}(32 \%), \mathrm{H}-2 \rightarrow \mathrm{L}+1(49 \%)$ \\
\hline
\end{tabular}

